\title{
GRYSTALLIZED STATE OF POLYMER IN ITS DISPERSION BEHAVIOUR
}

\author{
Motowo Takayanagi \\ Department of Applied Chemistry, Kyushu University, Fukuoka, Japan
}

INTRODUCTION

In the field of crystalline polymers the morphological and structural studies of polymers have remarkably developed since the fold structure of single crystal of polymers was discovered by Keller ${ }^{1}$ in 1957 . This discovery has rendered us a new structural view, which is contributing to the interpretation of physical properties of crystallized polymers.

Strict analysis of molecular arrangements within polymer crystal and beautiful electron photomicrographs of lamellar structure as observed in bulk crystallized linear polyethylene seem to admit no question of the state of aggregation of polymer molecules in the crystalline texture. This view is, however, not adequate in the case of polymers such as polyester, polyamide and polypropylene having $40-50$ per cent amorphous region in their bulkcrystallized texture. Such polymers are rather more frequently found than highly crystalline polymers such as high density polyethylene and polyoxymethylene. For the polymers with medium degree of crystallinity the structural studies using x-ray diffraction, electron photomicrography and other methods seem to be insufficient to describe the state of aggregation of molecules in the crystalline texture, especially in the amorphous region. Dispersion behaviour of polymers gives information different from the structural studies in some point in grasping the actual state of crystallized polymer. The main purpose of this article is to describe the real state of crystallized polymer by supplementing the information of structural studies with knowledge obtained from dispersion behaviours.

\section{AGGREGATION OF MOLEGULES AND DISPERSION PHENOMENA}

Molecular chains under less restraint in the crystalline texture initiate their thermal motions at a lower temperature or at a shorter time (higher frequency), while molecular chains under greater restraint initiate their thermal motion at a higher temperature or at a longer time. This means that the difference in the state of aggregation of molecules could be discussed by measuring the change of dispersion behaviour. Temperature dependence of relaxation time or activation energy for relaxation and shape of relaxation spectrum also allow us the more detailed discussion on the state of aggregation of molecules.

Relaxation mechanisms are detected by measuring dispersion or absorption phenomena. As for viscoelasticity the measurements of transient phenomena such as stress relaxation and creep are useful, while dispersion of storage modulus and absorption of loss modulus obtained by the dynamic 


\section{MOTOWO TAKAYANAGI}

method using periodic excitation are frequently more sensitive and effective to achieve the present purpose. When the average relaxation time $\bar{\tau}$ is assumed to be characteristic of some type of molecular motion, the initiation of this motion can be sensitively detected by observing the dispersion of storage modulus $E^{\prime}$ and the absorption of loss modulus $E^{\prime \prime}$, which appear under the condition that $\omega \bar{\tau}=1$ is satisfied by changing frequency at a definite temperature or by changing temperature, that is, by changing $\bar{\tau}$ at a definite frequency (isochronal measurement).

As a typical example of dispersion curve of the crystalline polymer, that of poly-4-methyl-pentene- 1 is cited in Figure $1^{28}$. From the high temperature

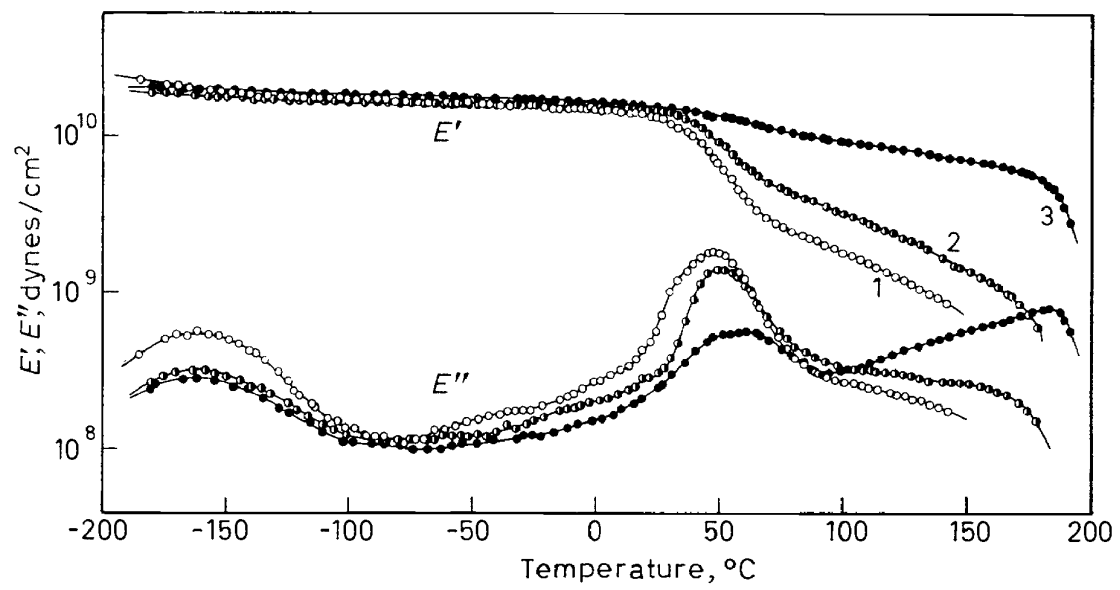

Figure 1. Tensile storage modulus $E^{\prime}$ and loss modulus $E^{\prime \prime} v s$. temperature at $110 \mathrm{c} / \mathrm{s}$ for three kinds of samples of isotactic poly-4-methyl-pentene-1 [(1) Bulk-crystallized sample quenched at $-70^{\circ} \mathrm{C}$ from the melt, (2) Annealed sample at $210^{\circ} \mathrm{C}$ for $15 \mathrm{~h}$, (3) The single crystal mat]

side, the crystalline absorption is found at about $170^{\circ} \mathrm{C}$, which increases its intensity with increasing crystallinity, the primary absorption associated with the initiation of the micro-brownian motion in the amorphous region is found at about $50^{\circ} \mathrm{C}$, which decreases its intensity with increasing crystallinity. The secondary absorption associated with the local twisting motion of the main chains is latent in the background of the primary absorption. Generally speaking, the secondary absorption of vinyl compounds absent from any flexible side chain such as isotactic polypropylene, polystyrene and polyvinyl alcohol are similarly latent, appearing only as a broad weak shoulder of the primary absorption, while the secondary absorptions of high density polyethylene and polyoxymethylene without side chains are usually very remarkable. The secondary absorption associated with the thermal motion of side chain is found at about $-160^{\circ} \mathrm{G}$ at $110 \mathrm{c} / \mathrm{s}$ distinctly for poly-4-methyl-pentene-1.

Crystalline texture will be roughly divided here into the crystalline and the amorphous region. Discussions will be given of each region referring to some recent works of the author's laboratory. In addition to these discussions, the mechanical coupling of the amorphous and the crystalline region will be taken up as another subject of this article. The change of dispersion be- 


\section{GRYSTALLIZED STATE OF POLYMER IN ITS DISPERSION BEHAVIOUR}

haviours caused by a different way of coupling of the two regions is considered to reflect the change of the superstructure of the crystallized polymer.

\section{DISPERSION ASSOCIATED WITH GRYSTALLINE REGION}

\section{Structurally defined polymer crystals and their dispersion behaviours}

As polymer single crystal has a high degree of crystallinity compared with that of the bulk crystallized polymer, the dispersion phenomena associated with the crystalline region can be remarkably observed for the single crystal sample 2,3 .

Measurements of viscoelasticity of single crystal were made in the form of the single crystal mat. Sinusoidal tensile strain was applied along the surface of single crystal mat. Measurements were made by using direct reading dynamic viscoelastometer, Vibron DDV-II of Toyo Measuring Instrument Co. Ltd, the principle of which was originated by our laboratory4. Tensile storage modulus $E^{\prime}$ and $\tan \delta$ at a definite frequency can be easily read off against temperature and loss modulus $E^{\prime \prime}$ was evaluated by $E^{\prime}$. $\tan \delta$. The detailed descriptions of sample preparation and measurement were given in the earlier papers $2,3,16$.

Crystalline absorption of the single crystal mat is affected by the morphological factors of crystal and its perfection. Single crystal samples of linear polyethylene with different thickness $L$ were prepared by strictly regulating the conditions of isothermal crystallization. Lamellar thickness $L$ was evaluated by the long period of x-ray small angle scattering. Figure 2 shows the systematic increase of intensity of the crystalline absorptions and the shift of the dispersion temperature towards the high temperature side with increasing lamellar thickness from 94 (curve 1) to $167 \AA$ (curve 5) ${ }^{5}$. By changing the frequency of measurements and applying the method of reduced variables the master curve of $E^{\prime \prime}$ is obtained and from this curve the relaxation spectrum can be evaluated with the usual approximate method 2 . Figure 3 shows the relaxation time spectra of the single crystal mats with lamellar thickness of 94,104,114, 137 and $167 \AA$. The average relaxation time $\bar{\tau}$ shifts to the side of longer relaxation time with increasing lamellar thickness. This tendency will be qualitatively understood by considering the corresponding modes of torsional motion of molecular segments constituting the different lamellar thickness. The more elaborated theory has been developed by Okano ${ }^{6}$. According to Okano's theory the tendencies of the increased intensity of relaxation and the increased absorption temperature with increasing thickness are expressed by the linear relationships of $\log L$ vs. reciprocal temperature of the crystalline absorption and of the average relaxation time vs. $L^{2}$. However, definite discrepancies were found between our experimental results and the relationships predicted by Okano. The shape of the relaxation spectra of the crystalline absorption is very broad when compared with that of the primary absorption of the completely amorphous polymer. The interpretation of such broad spectra by the molecular theory is a problem to be solved in the future, while the broadness is considered to be caused by the coupled oscillation of many molecular chain segments composing a crystal block in single crystal. 


\section{MOTOWO TAKAYANAGI}

It is well known that the single crystal of polyethylene thickens by heattreatment above its crystallization temperature. Figure 4 shows absorption curves for the annealed single crystal mats of polyethylene. With increasing lamellar thickness determined by long period of small angle x-ray diffraction, the absorptions of the samples shift to the high temperature side in the

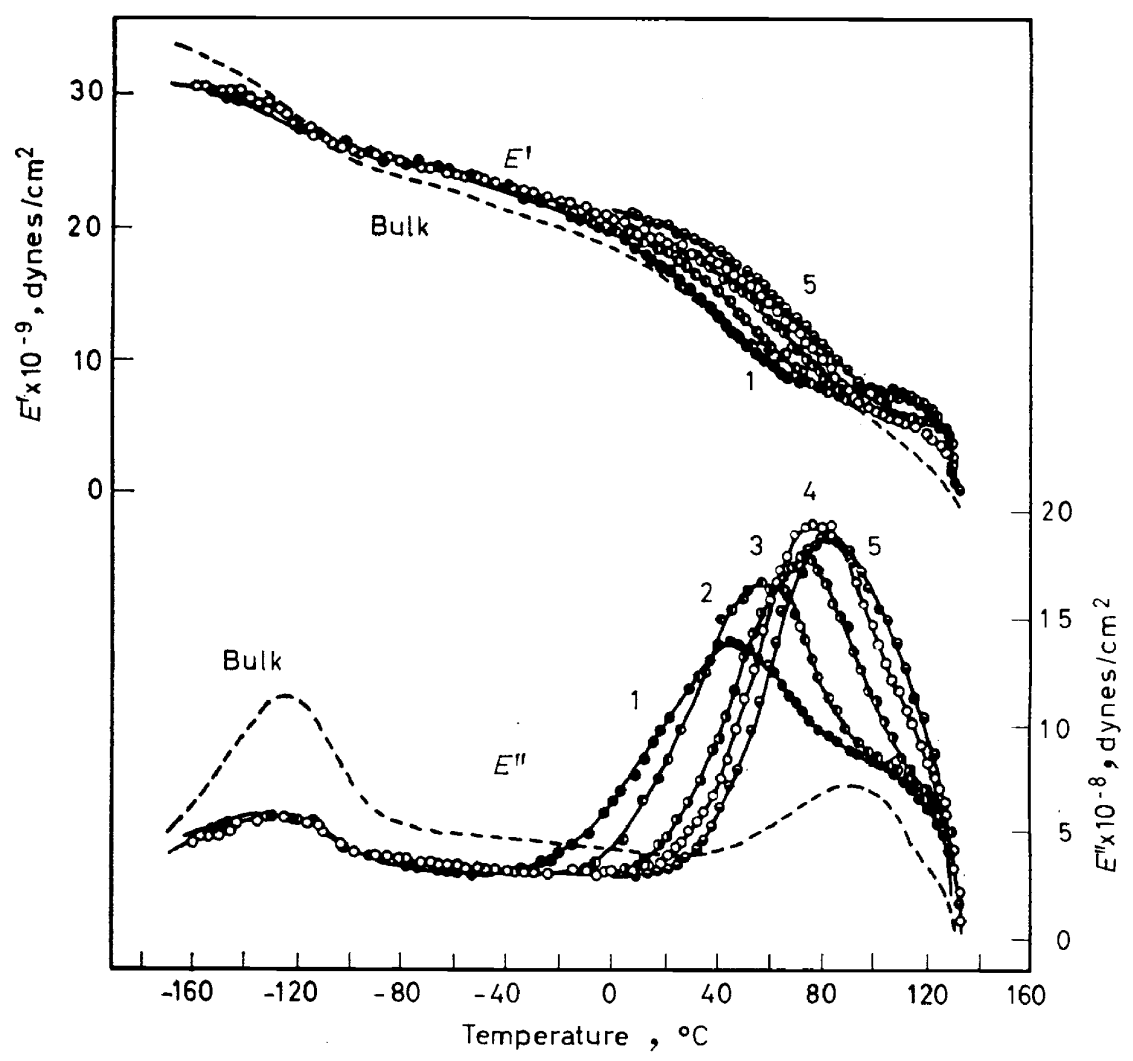

Figure 2. $E^{\prime}$ and $E^{\prime \prime} v s$. temperature at $110 \mathrm{c} / \mathrm{s}$ for the mats of single crystal of linear polyethylene (Marlex 50) with different thickness prepared at different crystallization temperatures from $0.06 \%$ xylene solution. No heat treatment [Curves $1,2,3,4,5$ correspond to the single crystal mats with long period values of $94,104,114,137$ and $167 \AA$ respectively; $(--)$ are for the bulk-crystallized sample]

same way as in the case of the isothermally crystallized samples. It should be noticed, however, that the absorption intensity of the crystalline absorption of the annealed samples decreases with increasing thickness contrary to the case of the isothermally crystallized samples. Figure 5 shows a plot of the absorption temperature $T_{\max }\left(\alpha_{\mathrm{c}}\right)$ against long period $L$. The relation is very similar to the relation of melting temperature of polyethylene crystal evaluated by Lauritzen and Hoffman ${ }^{7}$ or of n-paraffin evaluated by Broadhurst ${ }^{8}$ to $L$. The $T_{\max }\left(\alpha_{e}\right)$ of both the samples obtained by isothermal crystallization and by annealing is expressed by a single curve as a function of long period. On the other hand, the $E^{\prime \prime}{ }_{\max }$ values of the isothermally 


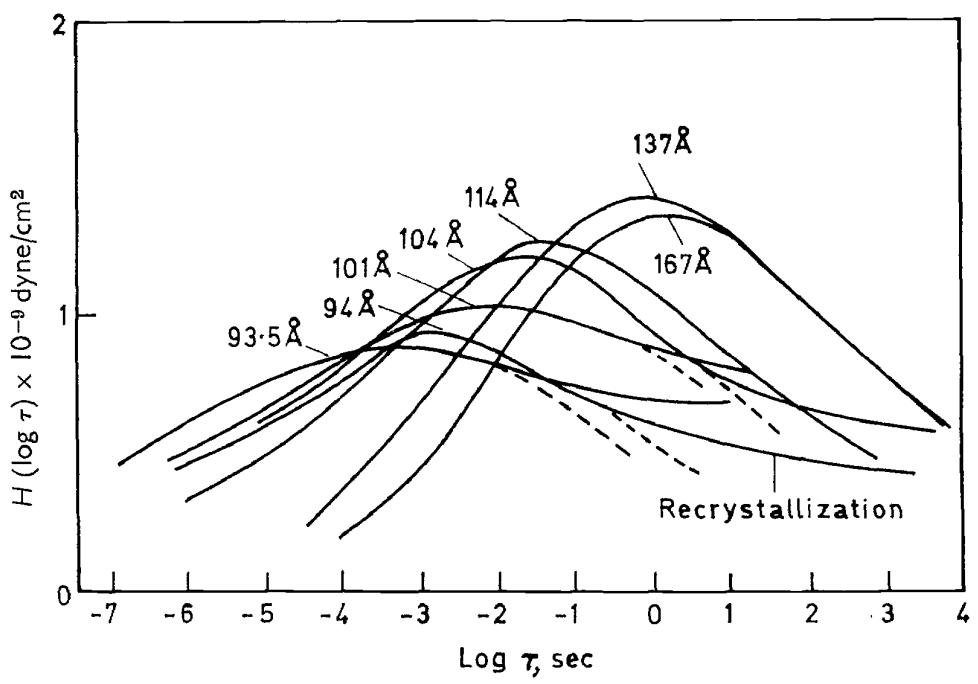

Figure 3. Relaxation spectra at $60^{\circ} \mathrm{C}$ for the single crystal mats with long periods of 94,104 , 114,137 and $167 \AA$ which are prepared from the composite curve of $E^{\prime \prime}(\omega)$ based on the measurements at different frequencies and temperatures

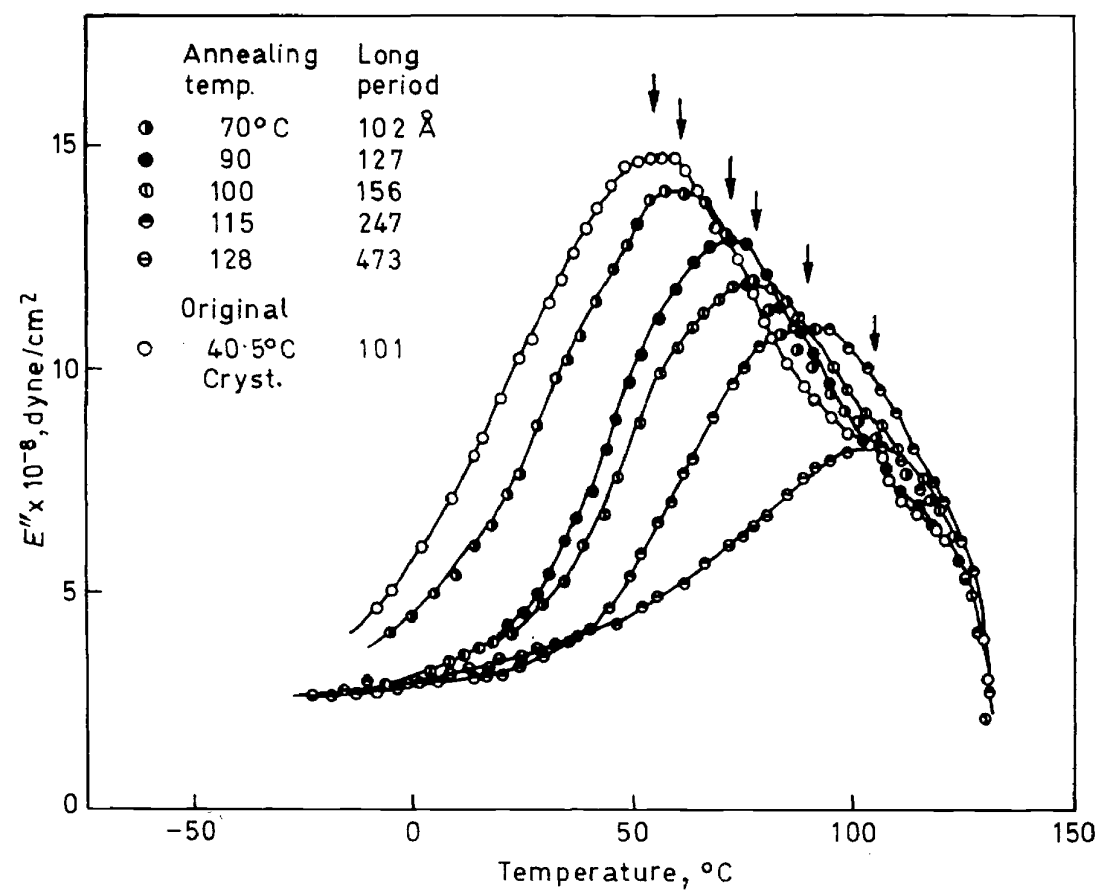

Figure $4 . E^{\prime \prime}$ vs. temperature at $110 \mathrm{c} / \mathrm{s}$ for the single crystal mats of linear polyethylene annealed at $70^{\circ}, 90^{\circ}, 100^{\circ}, 115^{\circ}$ and $128^{\circ} \mathrm{C}$, corresponding to the long periods of $102,127,156$, 247 and $473 \AA$. Original single crystal prepared at $40 \cdot 5^{\circ} \mathrm{C}$ is $101 \AA$ thick. 
MOTOWO TAKAYANAGI

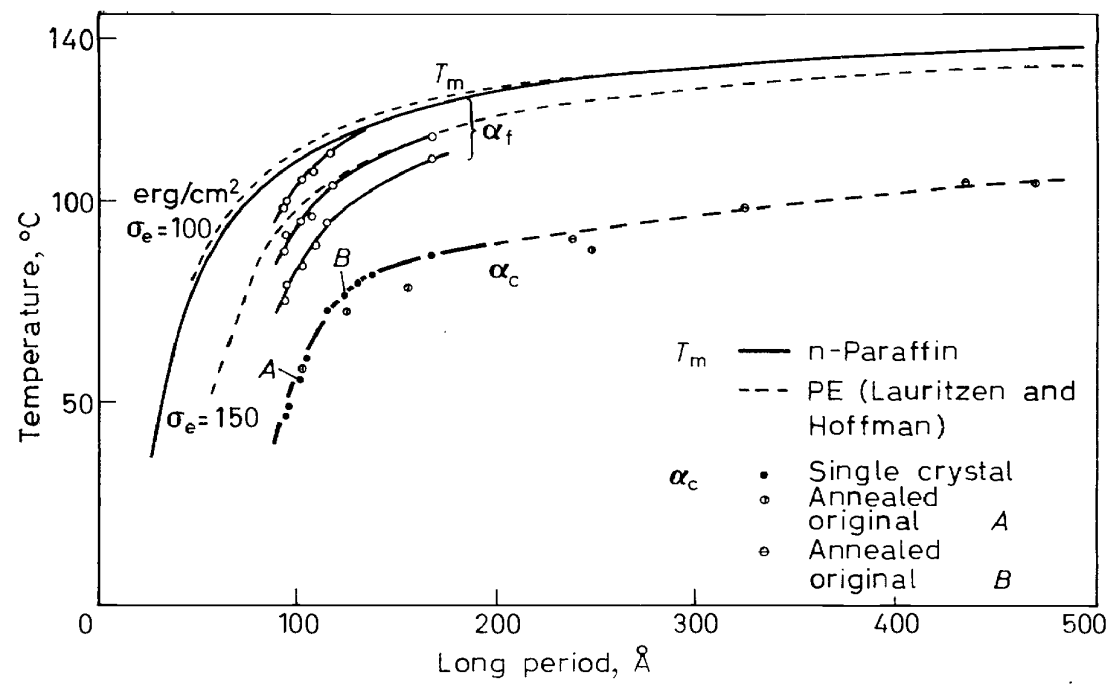

Figure 5. Temperature of the crystalline absorption $\left(\alpha_{c}\right)$ maximum $v s$. long period for the isothermally crystallized polethylene single crystal (O) and the annealed samples starting from the single crystals $A$ and $B\left((D, \Theta)\right.$. Melting temperature $T_{\mathrm{m}} v s$. long period (-- - for polyethylene single crystal after Lauritzen and Hoffman's equation. The crystalline absorption $\left(\alpha_{p}\right)$ temperature associated with the irreversible structural change ${ }^{15} v s$. long period for the isothermally crystallized samples (O).

crystallized samples and the annealed ones are not expressed by a single curve but by two separate curves of quite different types as shown in Figure 6 .

The only possible interpretation for these contradictory relationships of $T_{\max }\left(\alpha_{\mathrm{c}}\right)$ and $E^{\prime \prime}{ }_{\max }\left(\alpha_{\mathrm{c}}\right)$ against long period $L$ is that the value of $T_{\max }$ is primarily determined by the thickness of crystal with almost perfect

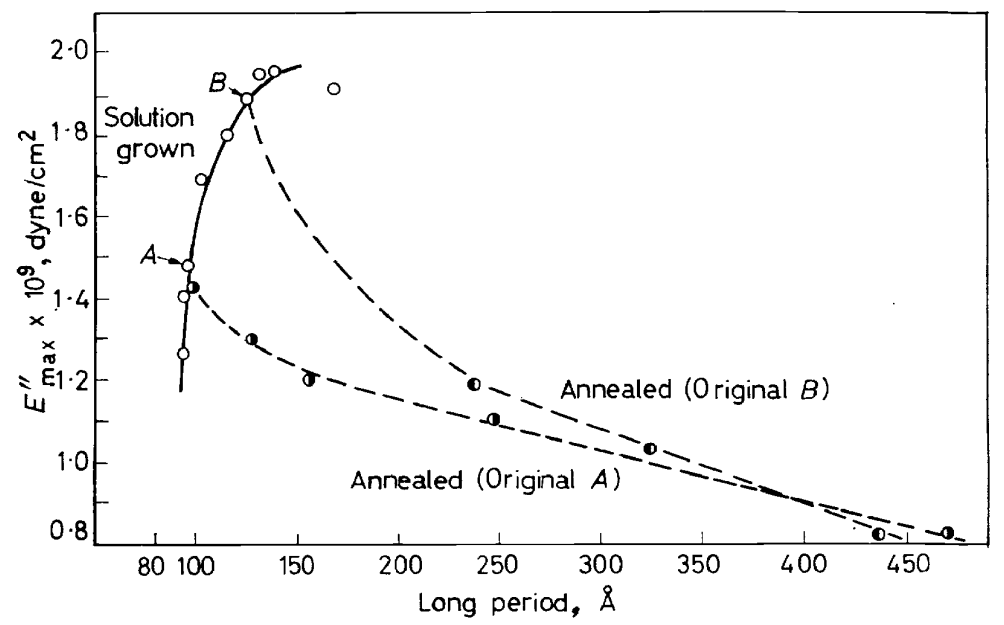

Figure 6. Maximum intensity of the crystalline absorption $E^{\prime \prime} \max v s$. long period for the isothermally crystallized samples $(\bigcirc)$ and the annealed samples $(\bigcirc, \mathcal{O})$. Results of two series of annealed samples are shown for the original crystals $A$ and $B$. 
regularity, but, on the other hand, the absorption intensity $E^{\prime \prime} \max \left(\alpha_{c}\right)$ is determined by the quantity of the crystal of the thickness $L$. If so, the quantity of such a perfect crystal region with thickness $L$ should be decreased by increased annealing temperature.

The support of this view from the structural or morphological studies is not enough to confirm the view. There are, however, some experimental facts concerned with it. According to Holland ${ }^{9}$ the moire pattern of the annealed polyethylene single crystal has a very much distorted aspect, indicating that the concentration of dislocation is increased by a factor of one thousand as compared with that of the original crystal. In such a case the crystal part with perfect regularity will be largely decreased. The $(110)$ plane used in their observation is considered to be distorted like an amoeba shape and the area showing the regular stripes as observed in the isothermally crystallized samples evidently decreased to a fraction of the original ones. Such a structural change will be enough to interpret the decrease of the absorption intensity with increasing $L$ as shown in Figure 6 . The nature of the defect region is also discussed by Kedzie ${ }^{10}$, who has found the fact that the narrow component of the differential curve of n.m.r. absorption of the single crystal shows a remarkable anisotropy, i.e. the second moment takes a maximum value when the magnetic field direction accords with that of molecular axis in the crystal. The broad component has two maxima depending on the orientation of the crystal. The fact that the narrow component has anisotropy means that the defect region shows preferable orientation along the direction of molecules in the crystal and that it exists within the crystal phase. We assume here that the defect regions are included in the lamellae thickened by heat-treatment and they do not contribute to the crystalline absorption. Slichter ${ }^{11}$ found that the narrow component appears by heat-treatment of single crystal of polyethylene and it increases its intensity with increasing rate by raising the temperature of annealing. This fact corresponds well with the observation that the $E^{\prime \prime}$ max of the crystalline absorption decreases with increasing annealing temperature.

It is known that the broad component of n.m.r. spectrum of the solution grown polyethylene crystal narrows at a certain temperature range with increasing temperature. According to Iwayanagi ${ }^{12}$ the temperature at which the broad component begins to narrow rises with increasing crystallization temperature. This means that the molecular motions in the crystal region of perfect regularity tend to initiate at the lower temperature for the thin crystal obtained at the low crystallization temperature. This tendency is in accordance with our observation that the $T_{\max }\left(\alpha_{c}\right)$ shifts to the high temperature side with increasing crystallization temperature as shown in Figure 4.

Sinnott ${ }^{13}$ has recently reported on the mechanical relaxations in single crystal mat of polyethylene; his results are almost the same as ours in that the crystalline absorption of the annealed and thickened crystal shows the decreased relaxation intensity (expressed by Sinnott with the absorption area instead of with the absorption maximum reported by us). Based on this observation Sinnott proposed that the crystalline absorption is associated with the folded region attached to the surface of the thickened lamella. This interpretation seems to encounter the difficulties in interpreting our 


\section{MOTOWO TAKAYANAGI}

observation that the crystalline absorption intensity increases with increasing long period for the isothermally crystallized samples with different thicknesses. Mechanical dispersions associated with the loose loops attached to the crystal surface will be discussed in more detail under the next section, discriminating from the crystalline absorption mentioned here.

In the above discussion the maximum intensity of the absorption $E_{\text {max }}^{\prime \prime}$ was employed for convenience as the measure of the magnitude of an absorption. The magnitude of dispersion $\Delta E$ is given by the following equation based on the interrelation of viscoelastic functions ${ }^{14}$.

$$
\Delta E=\int_{-\infty}^{\infty} H_{1} \mathrm{~d} \ln \tau=\frac{2}{\pi} \int_{-\infty}^{\infty} E^{\prime \prime}(\omega) \mathrm{d} \ln \omega .
$$

Evaluation of $\Delta E$ values of the isothermally crystallized samples was made using the relaxation spectra of Figure 3 and compared with the $E^{\prime \prime}$ max value of Figure 2. Satisfactory proportionality was obtained between both values of $E^{\prime \prime} \max$ and $\Delta E$ corrected for the recrystallization effect.

It should be noticed, however, that the absorption curves of the annealed single crystal and the bulk crystallized sample 2,15 could not be reduced to prepare the composite curves of $E^{\prime \prime}$ due to the appearance of the new crystalline absorption $\left(\alpha_{c}^{\prime}\right)$ in addition to the $\alpha_{c}$ absorption at its low temperature side ${ }^{15}$. We did not try here to evaluate these two absorptions separately. $E^{\prime \prime}{ }_{\max }$ value was employed as the measure of magnitude of an absorption in the above discussion.

Next we would like to proceed to the subject of crystalline texture of the bulk crystallized samples apart from the problem of single crystal. It is well known that the spherulite is composed of piled thin plate-like crystals (lamella.), which are very similar in their structure to the single crystal except an existence of tie molecules between neighbouring lamellae in the spherulite. The foregoing discussions on the single crystal will be, therefore, reasonably applied to the crystalline texture of the bulk crystallized sample under some restrictions.

Broken lines of Figure 2 are the $E^{\prime}$ and $E^{\prime \prime}$ vs. temperature curves at $110 \mathrm{c} / \mathrm{s}$ for the bulk crystallized polyethylene. Compared with the isothermally crystallized single crystal, the bulk crystallized sample rather resembles the annealed single crystal in the shape of its dispersion curves. According to our concept of the crystallized state of isothermally crystallized single crystal and the annealed one mentioned above, the lamellar phase of spherulite includes a somewhat large defect region, by which the intensity of the crystalline absorption is largely decreased. And at the same time the low temperature absorption at about $-120^{\circ} \mathrm{C}$ of the bulk crystallized sample is remarkably increased compared with that of the single crystal samples as if it compensates the decreased magnitude of the crystalline absorption. The $-120^{\circ} \mathrm{C}$ absorption is called here the $\beta_{\mathrm{a}}$ absorption, which is closely related to the amounts of defect region in the crystal and of the frozen amorphous region. The same relation can be found in the absorption curves of polyoxymethylene between the samples of the single crystal mat and the bulk crystallized film ${ }^{16}$. 


\section{CRYSTALLIZED STATE OF POLYMER IN ITS DISPERSION BEHAVIOUR}

The authors 17 have recently found an experimental support for the concept that the low temperature secondary absorption associated with side chain motion $\beta_{\mathrm{Sc}}$ (some authors call it $\gamma$ ) is divided into two components; the one is concerned with the frozen amorphous region $(\beta-\mathrm{A})$ and the other with the defect region in the crystalline phase $(\beta-\mathrm{C})$. The secondary absorption at about $-155^{\circ} \mathrm{C}$ of the bulk crystallized isotactic poly-4-methylpentene-1 (P4MP1) splits into two separable regions; the higher temperature one is concerned with the frozen amorphous region, its temperature location is in accordance with that of the completely amorphous atactic samples and its magnitude (the area under the $E^{\prime \prime} v s .1 / T$ curve) decreases in proportion to the fraction of the amorphous region or with increasing crystallinity $(\chi)$, and the lower temperature one is concerned with the defect region of the crystalline phase, although its magnitude (the area of the absorption separated from that of the frozen amorphous region) is not necessarily proportional to the degree of crystallinity $\chi$, but amounts to some fraction of $\chi$ depending upon the perfectness of crystalline region. It should be noted that the secondary absorption of P4MP1 at about $-155^{\circ} \mathrm{C}$ is concerned with the thermal motion of side chain $\left(\beta_{\mathrm{sc}}\right)$ and not with the local twisting motion of main chain $\left(\beta_{\mathrm{a}}\right)$ as mentioned in the introduction. The separation of the $\beta_{\mathrm{sc}}$ absorption of P4MP1 into the one associated with the frozen amorphous region $\beta_{\mathrm{sc}}-A$ and the defect region $\beta_{\mathrm{sc}}-C$ is illustrated in Figure 7 . The fraction of the amorphous region $(A)$ can be evaluated as the ratio of the area of the $\beta_{\mathrm{sc}}-A$ absorption to that of the atactic sample. Defect region $\left(A^{\prime}\right)$ can be evaluated by the value of $A$ multiplied by the ratio of the magnitude of $\beta_{\mathrm{sc}^{-}}-C$ absorption to that of the $\beta_{\mathrm{sc}}-A$ absorption. For example the bulk crystallized P4MP1 with degree of crystallinity $\chi=\mathbf{0 . 4 6}$ evaluated by the x-ray method gives the values of $A=0.56$ by the absorption method, the absorption ratio of $\beta_{\mathrm{sc}^{-}}-C / \beta_{\mathrm{sc}}-A=0 \cdot 28 / 0 \cdot 72$, and therefore $A^{\prime}=0.56 \times 0.28 / 0.72=0.22$. The fraction of perfect crystal $C$ is given by $1-A-A^{\prime}=0 \cdot 22$. The value of $A^{\prime} /(1-A)$, the fraction of the defect region in lamellar phase, is evaluated as 0.50 , i.e. the defect region amounts to about a half of lamella. The $A^{\prime} /(1-A)$ value depends upon the crystallization condition of the sample, and its low value corresponds to a high degree of perfection of crystal regularity. The case of P4MP1 is rather fortuitous, as its secondary absorption is separable into two regions. Usually two such regions are united into one secondary absorption.

Another evaluation method of defect region in the lamellar phase of the bulk crystallized sample was previously presented by the authors 18 in the case when the primary absorption (called here $\alpha_{a}$ ) associated with the initiation of micro-Brownian motion of the main chains belonging to the amorphous region can be found. This method is also based on the same concept of the secondary absorption as mentioned above. There are two kinds of secondary absorption; $\beta_{\mathrm{sc}}$ and $\beta_{\mathrm{a}}$. The latter is concerned with the local twisting motion of main chains and is often remarkable for the polymer absent from side chains. The treatment of $\beta_{\mathrm{a}}$ is the same as that of $\beta_{\mathrm{sc}}$.

The crystalline texture of the bulk crystallized polymer is divided again into three regions, $A, A^{\prime}$ and $C$, corresponding to the degree of thermal motion of molecules. The region $A$ is defined as the region, the molecules in which are capable of the micro-Brownian motion above its glass transition 


\section{MOTOWO TAKAYANAGI}

temperature. The region $A^{\prime}$ is defined as the region, the molecules in which are only capable of the local twisting motion ${ }^{19}$ but incapable of diffusional motion even if above $T_{\mathrm{g}}$. Such circumstances will be realized only within the lamellar phase. The region $C$ is the crystalline region with perfect

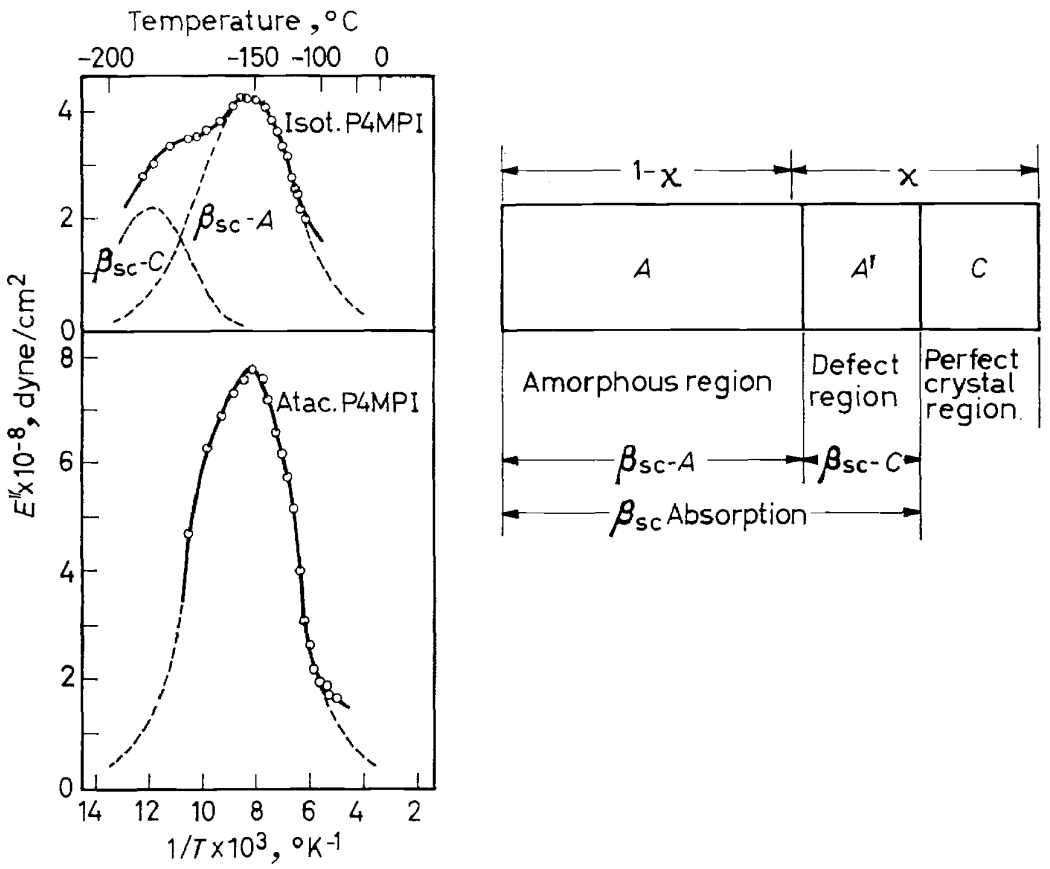

Figure 7. $E^{\prime \prime}$ vs. $1 / T$ at $110 \mathrm{c} / \mathrm{s}$ for the crystallized isotactic poly-4-methyl-pentene-1 (upper curve) and the atactic one in a temperature range of low temperature secondary absorption $\beta_{\mathrm{sc}}$. The secondary absorption $\beta_{\mathrm{sc}}$ is divided into two regions $\beta_{\mathrm{Bc}}-\mathrm{A}$ (high temperature absorption) and $\beta_{\mathrm{sc}}-\mathrm{C}$ (low temperature one).

The right side is the diagram representing the relationship among the degree of crystallinity $\chi$, the region $\mathrm{A}$ evaluated with the $\beta_{\mathrm{sc}} \mathrm{A}$ absorption, the region $A^{\prime}$ with the $\beta_{\mathrm{se}}-\mathrm{C}$ absorption, and the perfect crystal region $C=1-A-A^{\prime} . A$ is the fraction of the frozen amorphous region and $A^{\prime}$ is the defect region in lamella. Confer the evaluation method in the text.

regularity and the thermal motion associated with this region will be initiated at the temperature region of the crystalline absorption. According to these concepts the fractions of the $A, A^{\prime}$ and $C$ regions can be evaluated by the

$$
\begin{aligned}
& A=(\Delta E)_{\alpha} /(\Delta E)_{\alpha}^{\circ} \\
& A^{\prime}=(\Delta E)_{\beta} /(\Delta E)_{\beta}^{\circ}-A \\
& C=1-A-A^{\prime}
\end{aligned}
$$

relationships (1), (2) and (3), where $\Delta E$ is the magnitude of dispersion, and the denominator represents the quantity referring to the wholly amorphous sample and is evaluated by extrapolating the absorption magnitude to 
the zero crystallinity. Figure 8 schematically represents the relation among degree of crystallinity $\chi$ determined by physical methods such as density or x-ray, and the viscoelastically defined regions, $A, A^{\prime}$ and $C$.

Polyethylene terephthalate is a polymer suitable for application of the above method, since this polymer displays a remarkable $\alpha_{\mathrm{a}}$ absorption and a $\beta_{\mathrm{a}}$ absorption at the same time. The author ${ }^{\mathbf{1 8}}$ employed the $\alpha_{\mathrm{a}}$ absorption maximum $E^{\prime \prime}{ }_{\max }$ instead of $\Delta E_{\alpha}$ in Eq. (1). Illers ${ }^{20}$ has pointed out that the absorption magnitude should be evaluated by the area under the $E^{\prime \prime} v$. $1 / T$ curve. More detailed inspection of the $\alpha_{\mathrm{a}}$ absorption has been made by the author ${ }^{16}$ based on the relaxation spectra for the samples with different crystallinity. The shape of the relaxation spectra in a primary absorption region of the crystallized sample is not simple and suggests some composite relaxation mechanisms. At present the $\alpha_{\mathrm{a}}$ absorption magnitude can be more strictly evaluated in the dielectric absorption by using the area under the $\epsilon^{\prime \prime}$ vs. $\log f$ curve. The magnitudes of $(\Delta E)_{\alpha}$ and $(\Delta E)_{\beta}$ were plotted against degree of crystallinity $\chi$ for various kinds of polyesters 21,22 . The $\alpha_{a}$ absorption decreases in magnitude remarkably with increasing $\chi$, while the magnitude of $\beta_{\mathbf{a}}$ absorption does not decrease so much even when extrapolated at $\chi=1$. Figure 8 shows the schematic representation of the

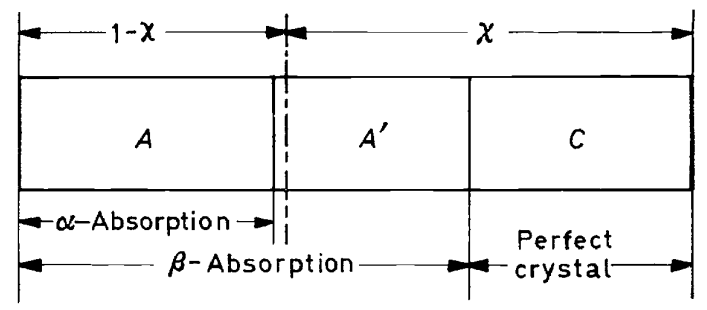

Figure 8 . Schematic representation of the relationship among degree of crystallinity $\chi$, the viscoelastically evaluated regions, $A, A^{\prime}$ and $C$ for the bulk crystallized polyethylene terephthalate with $\chi=0 \cdot 60$. For evaluating the regions $A, A^{\prime}$ and $C$ the magnitudes of the primary $\left(\alpha_{\mathrm{a}}\right)$ and the secondary $\left(\beta_{\mathrm{a}}\right)$ absorption were used. Related domains of these absorptions are indicated in the figure.

fractions of $A, A^{\prime}$, and $C$ regions of polyethylene terephthalate with $\chi=0 \cdot 60$. Referring to these evaluations, an assignment of the regions $A, A^{\prime}$ and $C$ to the real structure of spherulite will be made as shown in Figure 9. The region $A^{\prime}$ is assigned to the defect region within the lamella and the region $A$ is assigned to the amorphous region segregated from the lamellar phase. The model of the lamellar structure, which includes a large defect region depending on circumstances amounting to about a half of the lamellar phase, does not seem to be in conflict with our concept on the structure of the annealed and thickened single crystal.

At last the effect of the chemical structure of polymers upon the crystalline absorption will be discussed ${ }^{3}$. Single crystal mats of various polymers such as poly- $\alpha$-olefins, polyethers and polyvinylalcohol give the remarkable crystalline absorptions on the contrary to the bulk crystallized samples prepared from the same polymers. Figure 10 shows the temperature dependence of $\tan \delta$ at $138 \mathrm{c} / \mathrm{s}$ for the two kinds of solution-grown samples and the bulk-crystallized sample of isotactic polybutene-123. In the single crystal 


\section{MOTOWO TAKAYANAGI}

samples the primary absorption at about $0^{\circ} \mathrm{C}$ is remarkably depressed to be detectable only as a shoulder and the prominent crystalline absorption newly appears at $80^{\circ}$ or $100^{\circ} \mathrm{C}$ depending on the conditions of crystallization.

Activation energies $\Delta H^{*}$ evaluated by the temperature dependence of the crystalline peak position at different frequencies for several polymers, activation volumes $\Delta V^{*}$ and their melting temperatures $T_{\mathrm{m}}$ are listed in

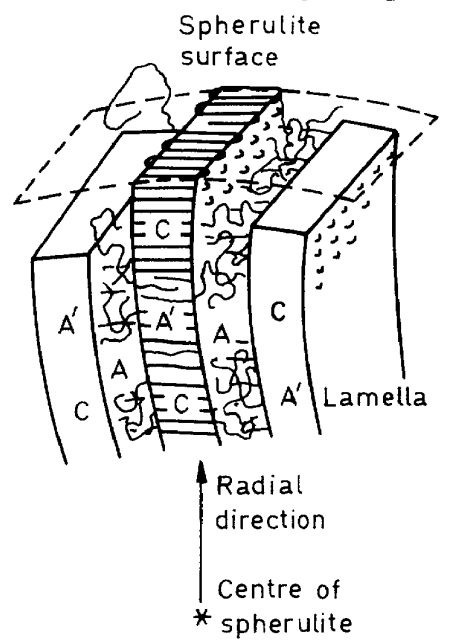

Figure 9. Model for the local structure of spherulite. The amorphous region $A$ is the region segregated from the lamellar phase, the region $A^{\prime}$ is the defect region in the lamellar phase and the region $C$ is the lamellar phase with perfect crystal regularity.

Table $1^{3}$. It should be noted that the value of PVA is considered to correspond to the unstable crystal form like smectic or nematic structure ${ }^{24}$. The solutioncast film was used for PVA sample.

The fact that the activation energy for relaxation of the crystalline region increases with increasing melting temperature leads us to the conclusion that the factors affecting the molecular motion associated with the crystalline region are common to those factors determining the thermodynamical stability of the corresponding crystals.

The fact that the typical crystalline absorption is closely related to the melting temperature concerned with the chemical structure and at the same time with the morphological factor strongly suggests that the crystalline absorption is associated with mechanical relaxation of the crystalline region with high regularity.

\section{Structurally undefined low order crystals and their dispersion behaviours}

There is a possibility of existence of a phase with intermediate regularity between the well-defined crystalline region and the amorphous region. Such a phase is usually difficult to describe crystallographically.

Figure 11 shows the $\tan \delta$ vs. temperature curves of the four kinds of samples of Nylon 6. In the solution grown crystal of Nylon 6 there is in its temperature dispersion curve a broad absorption at about $120^{\circ} \mathrm{C}$ at $110 \mathrm{c} / \mathrm{s}$ in addition to the typical crystalline absorption found at about $200^{\circ} \mathrm{C}^{3}, 25$. 


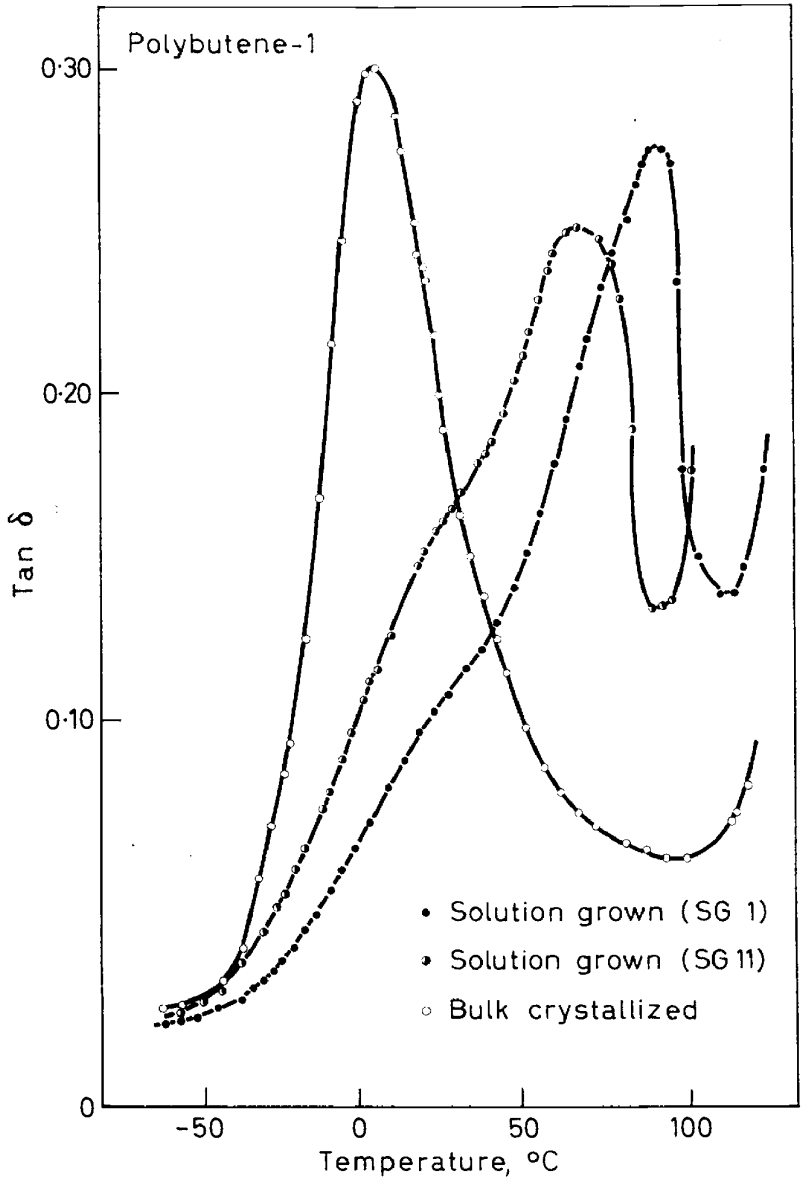

Figure 10. Tan $\delta$ vs. temperature at $138 \mathrm{c} / \mathrm{s}$ for the bulk crystallized sample $(0)$ and the solution grown samples ( and $)$ for isotactic polybutene-1

Table 1. Activation energies $\left(\Delta H^{*}\right)$, activation volumes $\left(\Delta V^{*}\right)$, and number of chain atoms $(N)$ concerned with crystalline relaxation

\begin{tabular}{|c|c|c|c|c|c|c|}
\hline Polymer & $\begin{array}{c}\Delta H^{*} \\
(\mathrm{kcal} / \mathrm{mole})\end{array}$ & $\begin{array}{c}T_{\mathrm{c}} \\
\left({ }^{\circ} \mathbf{C}\right)\end{array}$ & $\begin{array}{c}T_{\mathbf{m}} \\
\left({ }^{\circ} \mathbf{C}\right)\end{array}$ & $\underset{(\mathrm{ml} / \mathrm{mole})}{\Delta V^{*}}$ & $\begin{array}{c}V \\
(\mathrm{ml} / \mathrm{mole})\end{array}$ & $N=\frac{\Delta V^{*}}{V}$ \\
\hline PB-1 & & 70 & $97^{c}$ & $\mathbf{5 2 0}$ & $29 \cdot 4^{\mathrm{d}}$ & 18 \\
\hline $\mathrm{PE}$ & $30^{a}\left\{\begin{array}{l}16 \cdot 2^{b} \\
43 \cdot 7^{b}\end{array}\right.$ & 90 & 135 & $550\left\{\begin{array}{l}300 \\
808\end{array}\right.$ & $13 \cdot 9$ & $40\left\{\begin{array}{l}22 \\
58\end{array}\right.$ \\
\hline PVA & 48 & 130 & - & 800 & $16 \cdot 4$ & $49^{(00}$ \\
\hline PP & 48 & 120 & 165 & 820 & $22 \cdot 4$ & 37 \\
\hline POM & 55 & 160 & 180 & 860 & $9 \cdot 9$ & 87 \\
\hline
\end{tabular}

a $\Delta H^{*}$ is evaluated from the change of absorption maximum with frequency.

b $\Delta H^{*}$ is evaluated from the temperature dependence of shifting factor $a_{\mathbf{T}}$.

c Melting temperature of modification III of PB-1 determined by DTA method.

d $V$ is evaluated by the lattice constants of Mod III obtained by the authors; tetragonal, $a=b=14 \cdot 68 \AA$, $c=12 \cdot 61 \AA, 7_{2}$ helix. 


\section{MOTOWO TAKAYANAGI}

The $200^{\circ} \mathrm{C}$ absorption cannot be usually detected for the bulk crystallized samples of Nylon 6 . In the bulk crystallized samples the remarkable primary absorption (the $\alpha_{a}$ absorption) appears at about $80^{\circ} \mathrm{C}$ instead of the broad $120^{\circ} \mathrm{C}$ absorption of the solution-grown crystal. According to our view, the $120^{\circ} \mathrm{C}$ absorption of the bulk crystallized sample is considered to be still latent and unseparable, being in the high temperature side of the $\alpha_{a}$ absorption. This tendency can be more clearly seen in the $\tan \delta$ curve of the bulk crystallized sample in Figure 11 prepared by melting it at $300^{\circ} \mathrm{C}$, crystallizing

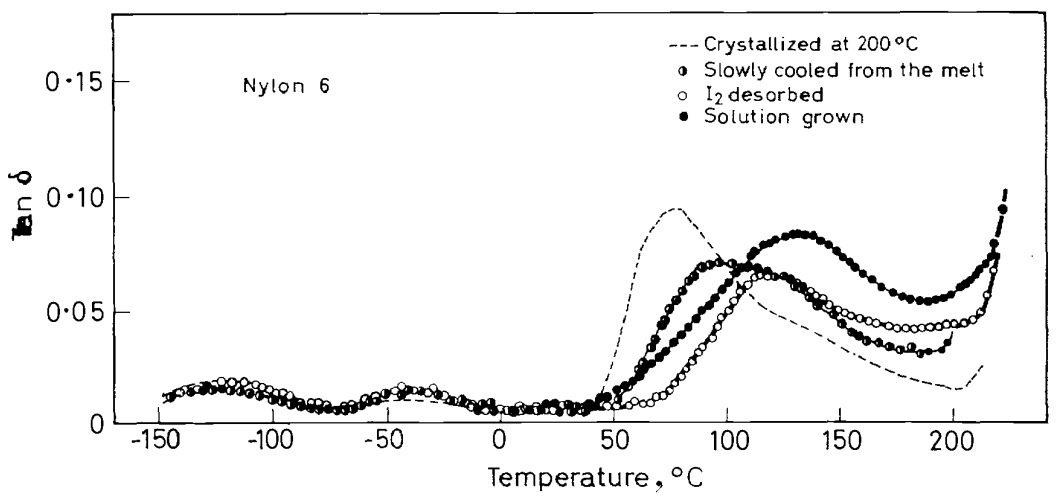

Figure 11. Tan $\delta$ vs. temperature at $110 \mathrm{c} / \mathrm{s}$ for four kinds of samples of Nylon 6 [(- - $)$ sample bulk crystallized at $200^{\circ} \mathrm{C}$, (O) sample prepared by slow cooling from the $300^{\circ} \mathrm{C}$ melt and removing generated $\epsilon$-caprolactum, $(\bigcirc)$ sample treated by iodine-iodide solution and desorbed by thiosulphate solution, and (1) mat of solution grown crystals prepared from the glycerine solution.

it slowly at a high temperature close to the melting temperature, and removing about 19 per cent of produced $\epsilon$-caprolactam by hot water. In this sample it can be shown that the $120^{\circ} \mathrm{C}$ peak coexists with the $80^{\circ} \mathrm{C}$ absorp. tion.

In even Nylons such as Nylon 6, 8 and $12^{25}$, when they are treated by iodine-potassium iodide solution and the iodine absorbed is rapidly removed by treating with alcoholic sodium thiosulphate solution, the dispersion curve similar to that of the solution grown sample is obtained, i.e. the $\alpha_{\mathrm{a}}$ absorption at about $80^{\circ} \mathrm{C}$ is largely depressed and the $120^{\circ} \mathrm{C}$ absorption remarkably increased as seen in the open circle curve in Figure 11. In this case iodine acts at first to break the hydrogen bonds in crystal and rapid desorption of iodine rebuilds the hydrogen bonds in a somewhat random way. This process seems to be closely related to the appearance of the $120^{\circ} \mathrm{C}$ peak for Nylon 6. The behaviours of Nylon 8 and 12 are very similar to those of Nylon 6.

On the other hand the odd Nylons such as Nylon 7, 9 and 11 give only the primary absorption at about $70^{\circ} \mathrm{C}$ without any background portion at its high temperature side even if the iodization and desorption treatment have been carried out ${ }^{25}$. Figure 12 shows the $\tan \delta v$ s. temperature curve of Nylon 9. It should be mentioned that any structural evidence using $x$-ray diffraction or infrared absorption cannot be found at present for the existence of the phase characteristic of the $120^{\circ} \mathrm{C}$ absorption. It is regrettable that the 
authors are still at the stage of speculation for interpreting these problems. One possible interpretation of the existence or absence of the $120^{\circ} \mathrm{C}$ absorption in even and odd Nylons will be presented next.

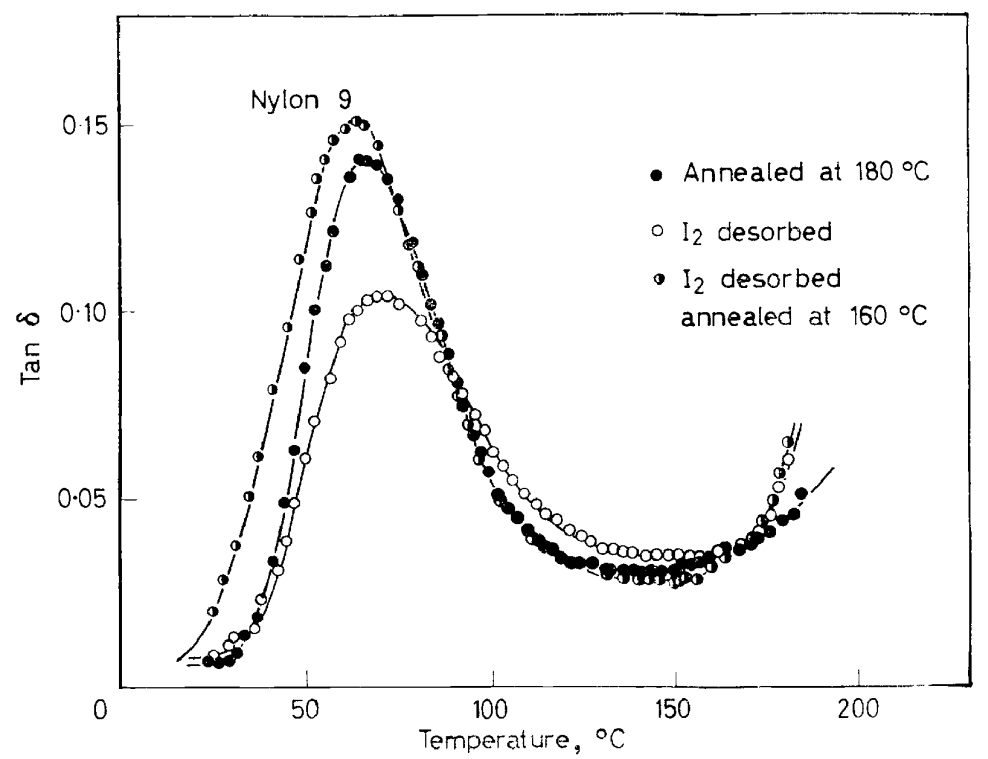

Figure 12. Tan $\delta$ vs. temperature at $110 \mathrm{c} / \mathrm{s}$ for three kinds of samples of Nylon 9 [( $)$ bulk crystallized sample annealed at $180^{\circ} \mathrm{G},(\bigcirc)$ iodine-adsorbed and desorbed sample, and

(O) is for the same sample annealed at $160^{\circ} \mathrm{G}$ after iodine is desorbed]

Even Nylon has a special crystal form or $\gamma$, which is composed of shortened fibre period to saturate all the hydrogen bonds between neighbouring parallel-directioned molecules, in addition to the usual crystal forms of $\alpha$ and $\beta$, which are composed of planar zigzag chains to saturate the hydrogen bonds between the anti-parallel directioned molecules. On the other hand odd Nylons have no necessity of assuming the $\gamma$ form in addition to the normal $\alpha$ and $\beta$ crystal forms, since both parallel-directioned and antiparallel-directioned molecules can completely saturate their hydrogen bonds between neighbouring molecules in an extended planar zigzag form. From such structural factors, if iodine is removed rapidly from the iodized samples, there is a possibility that for even Nylons both parallel and antiparallel-directioned molecules will form hydrogen-bonds in a mixed state, where the different fibre periods disturb the formation of the well defined crystal modification such as $\alpha$ or $\gamma$ form and as its consequence a phase of low regularity such as nematic structure will be formed. On the other hand for odd Nylons there will be no possibility of formation of nematic structure. The nematic form will behave almost in the same way as in the so-called amorphous region, while somewhat higher regularity of the nematic region will be enough to shift its absorption peak towards the high temperature side of the primary absorption.

It is worth mentioning that Nylon 66 has a possibility of formation of nematic structure in spite of the absence of $\gamma$ modification ${ }^{25}$. In the case of 


\section{MOTOWO TAKAYANAGI}

Nylon 66 there are two ways of arrangement of neighbouring planar zigzag molecules in the hydrogen bonded sheet: one way is to saturate all the hydrogen bonds between neighbouring molecules, and another way is realized by shifting the one planar zigzag chain along the neighbouring one by an amount of one residual radical, as the consequence of which only one half of the hydrogen bonds in the former case is saturated. Figure 13 shows
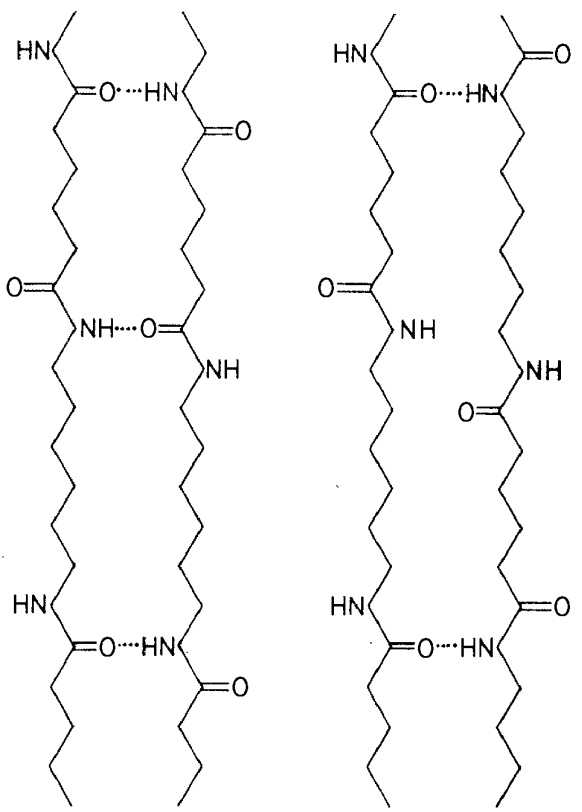

Figure 13. Two kinds of possibilities for hydrogen bond formations between two neighbouring Nylon 66 molecules. The right one is cited to interpret the possibility of forming of nematic structure in Nylon 66.

two kinds of arrangements of molecules in Nylon 66. These two molecular arrangements will be realized in a mixed state to form the nematic structure when the regeneration from the iodized state is rapidly processed. Figure 14 shows that the iodine-desorbed Nylon 66 has an increased absorption at about $150^{\circ} \mathrm{C}$, while the crystallized sample from the melt gives only a large primary absorption at about $60^{\circ} \mathrm{C}$ as is well known.

Polyvinyl alcohol (PVA) has an absorption at about $120^{\circ} \mathrm{C}$, which locates between the primary absorption at about $80^{\circ} \mathrm{C}$ and the crystalline absorption at about $200^{\circ} \mathrm{G}$. These absorptions are found in the solutioncast film ${ }^{24}$. The crystalline absorption is remarkable especially for the annealed solution-grown crystal ${ }^{3}$. The $120^{\circ} \mathrm{C}$ absorption increases its intensity for the samples partially acetylated or partially formalized as shown in Figure 1524. Such treatments will increase the nematic region in the crystalline texture of PVA.

Natta ${ }^{26}$ reported the smectic modification of isotactic polypropylene (PP) of pseudo-hexagonal form. The authors 27 have found in the dispersion curve of the quenched samples of PP the absorption characteristic of smectic 


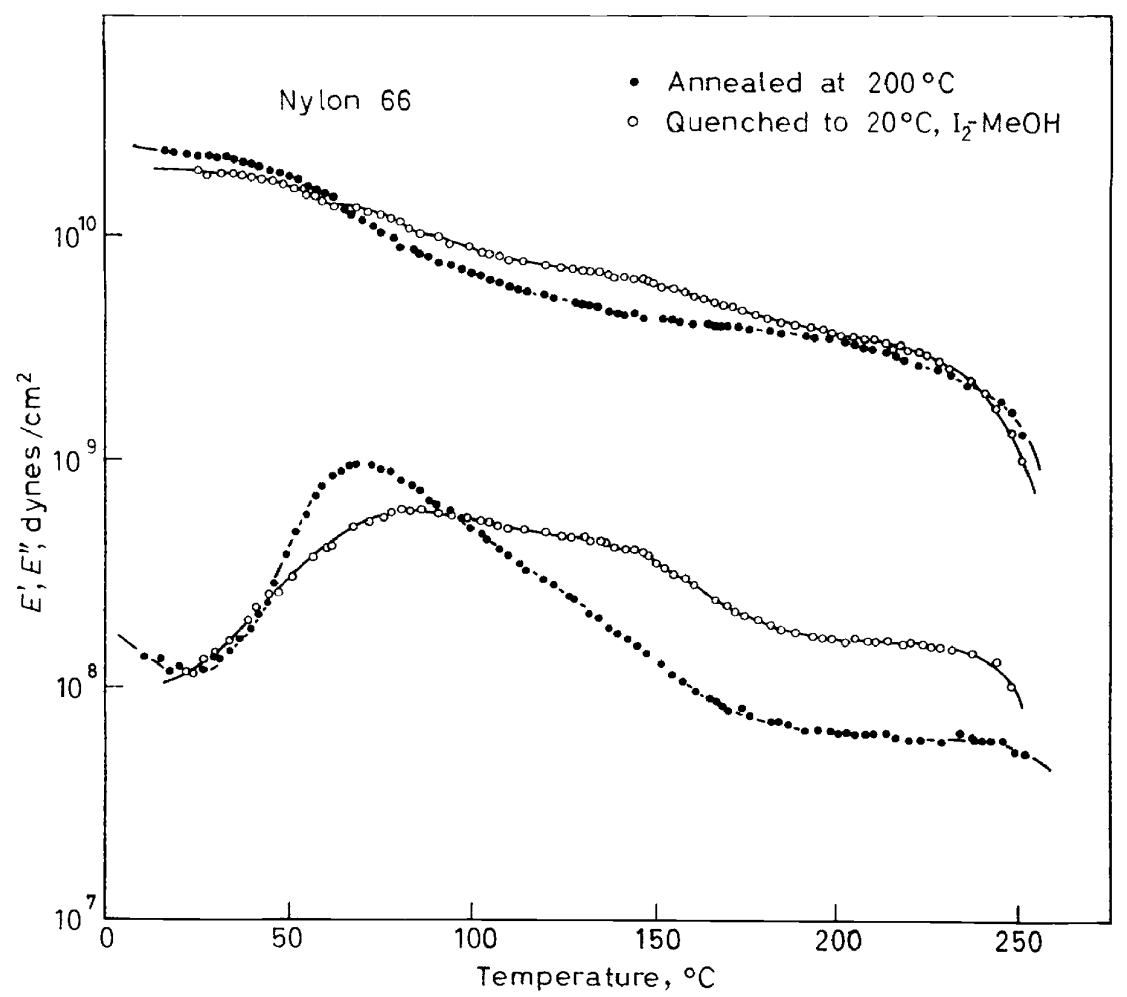

Figure 14. $E^{\prime}$ and $E^{\prime \prime}$ vs. temperature for the two kinds of samples of Nylon $66[(\mathcal{O})$ bulk crystallized sample, $(O)$ quenched sample, which is treated by iodine-iodide solution and then desorbed by methyl alcoholic thiosulphate solution. Notice the increased $150^{\circ} \mathrm{C}$ absorption]

structure at about $50^{\circ} \mathrm{C}$, being distinctly separated from the primary absorption locating at $20^{\circ} \mathrm{C}$ at $110 \mathrm{c} / \mathrm{s}$. Figure 16 shows the effect of treatment on the appearance of the $50^{\circ} \mathrm{C}$ absorption characteristic of smectic modification. Dilatometry proves that the melting temperature of the smectic structure is $50^{\circ} \mathrm{C}$. The process of remelting the smectic structure just above $50^{\circ} \mathrm{C}$ was reflected in an increase of the primary absorption, and the process of recrystallization by heat-treatment of the remelted sample was reflected in disappearance of the $50^{\circ} \mathrm{C}$ absorption being accompanied by a decrease of the primary absorption at $20^{\circ} \mathrm{C}$ together with an increase of crystallinity. Structural change of these processes studied by $\mathrm{x}$-ray diffraction well accorded with the change of absorption curves. The crystalline absorption of PP can be found at about $120^{\circ} \mathrm{C}$, especially distinctly for the solutiongrown crystals 27 .

The absorption associated with the unstable modifications are also observed for the solution grown crystals of poly-4-methyl-pentene- 1 at about $120^{\circ} \mathrm{C}$ as shown in Figure $17^{28}$, for the solution grown crystals of poly-npentene- 1 at about $50^{\circ} \mathrm{C}^{29}$, for the single crystal of poly-3,3-bischloromethyl oxacyclobutane at about $60^{\circ} \mathrm{C}$ while its $\alpha_{a}$ absorption is found at $30^{\circ} \mathrm{C}^{30}$. 


\section{MOTOWO TAKAYANAGI}

The crystal structure characteristic of these absorptions could not be found out crystallographically, while the regions characteristic of these absorptions are clearly different from those associated with the primary absorption. Such a structurally undefined but somewhat more ordered region than the

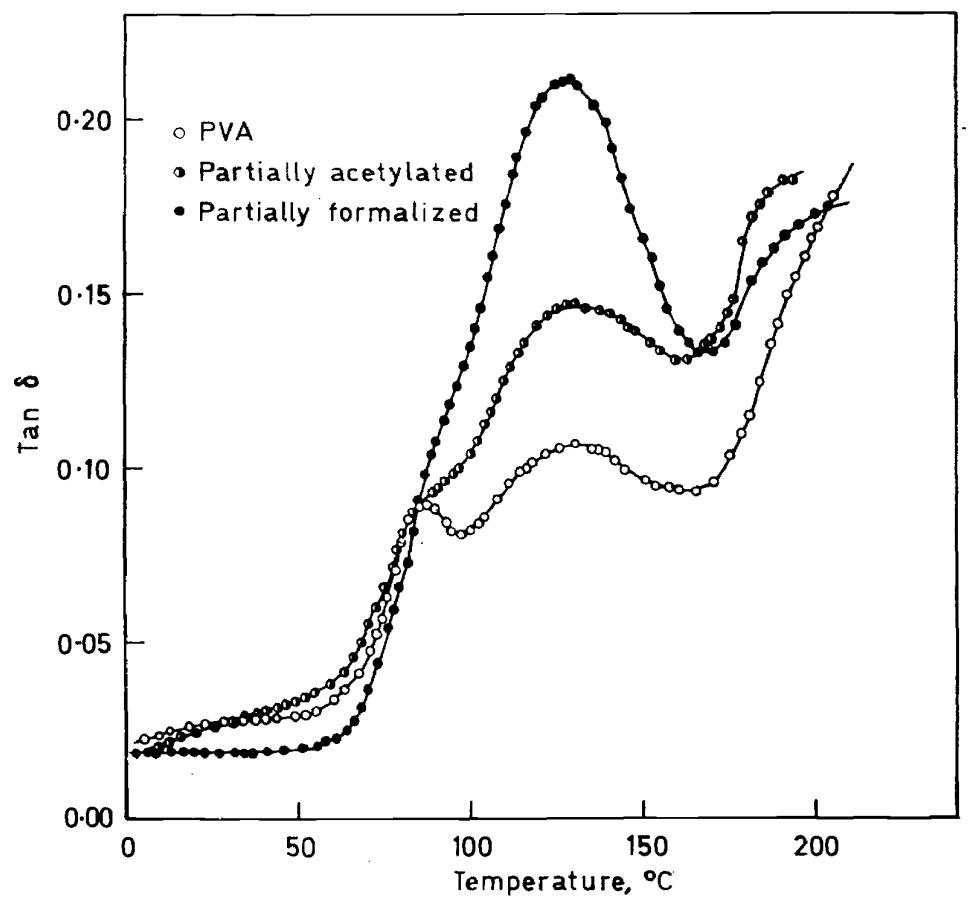

Figure 15. Tan $\delta v s$. temperature at $138 \mathrm{c} / \mathrm{s}$ for the solution-cast polyvinyl alcohol film (O), the partially acetylated film $(\mathbf{O})$, and the partially formalized film $(\mathbf{O})$, in a temperature range of primary absorption

amorphous region is to be taken into account to understand the actual crystallized state of polymers.

\section{DISPERSION ASSOGIATED WITH AMORPHOUS REGION}

The reasoning that the state of the amorphous region of the bulk crystallized polymer is different from that of the completely amorphous polymer will be partly supported by the fact that the relaxation spectrum associated with the primary absorption is always much broader than that of the amorphous polymer. Typical example is found in quenched and annealed polyethylene terephthalate, the degree of crystallinity of which can be regulated from 5 to 45 per cent ${ }^{16}$. The broadness of the spectrum is considered to be caused by the circumstances that the molecules within the amorphous region of the crystallized polymer suffer from various degrees of restraint by the surrounding lamellae.

The reasoning that the molecules causing the primary absorption are in a state of being segregated from the lamellar phase will be supported by the 
fact that the primary absorption of the crystallized isotactic polypropylene (PP) locates at almost the same temperature as that of the isotactic PP blended with atactic PP2,16. Atactic PP in the crystalline texture of the blend cannot be structurally incorporated into lamellae mainly composed of isotactic PP. The fact that the primary absorptions of both the samples resemble each other except their shapes means that the amorphous region of isotactic PP is not so much different from the state of atactic PP segregated from the lamellae in the blend system. Quantitative analysis based on this assumption has been given in the previous papers ${ }^{2}, 16$.

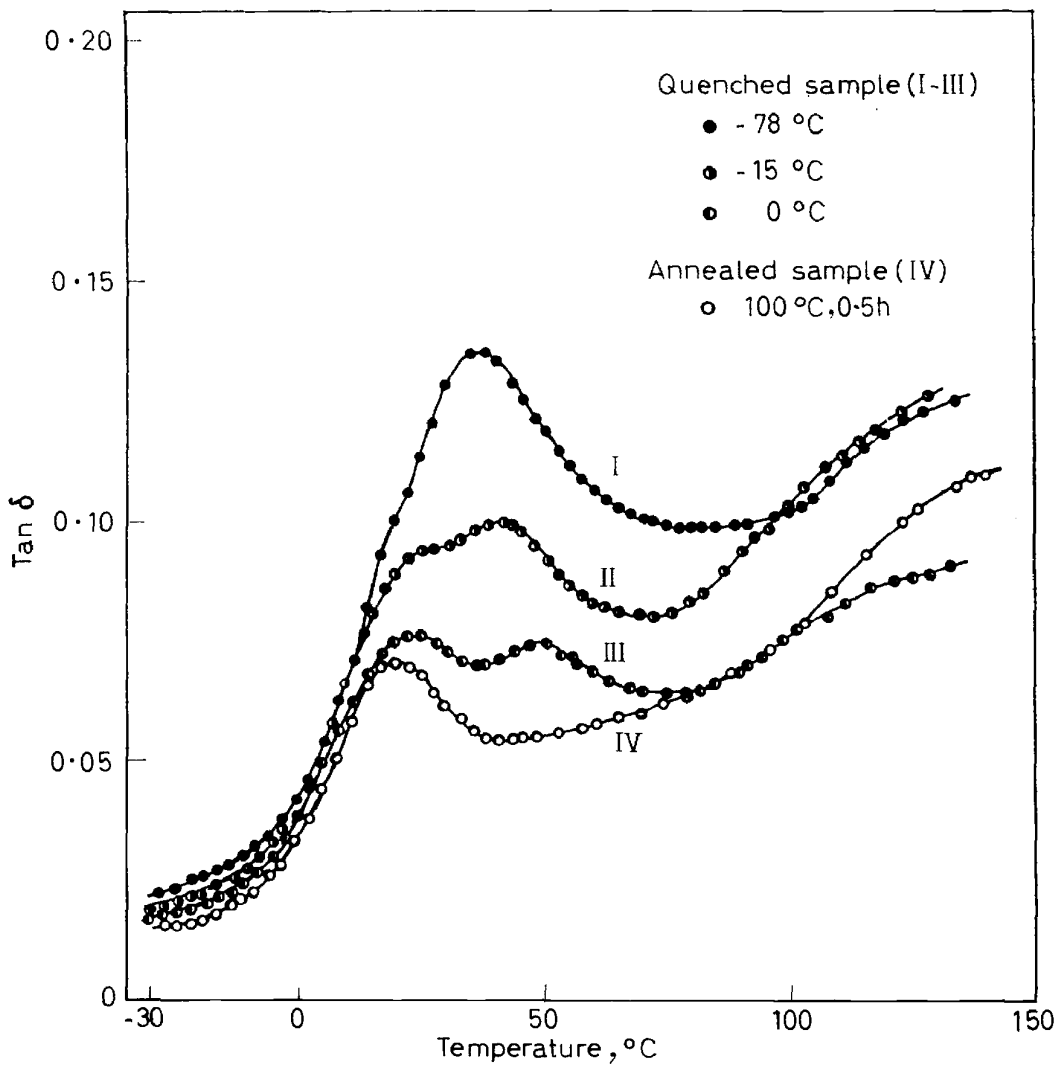

Figure 16. Tan $\delta$ vs. temperature at $110 \mathrm{c} / \mathrm{s}$ for the quenched and the annealed isotactic polypropylene samples. The primary absorption is found at about $20^{\circ} \mathrm{C}$ and the absorption concerned with smectic structure is at about $50^{\circ} \mathrm{C}$.

Theoretical considerations on the primary dispersion mechanisms seem to support the foregoing concept of the amorphous region. The primary absorption is related to the initiation of the micro-Brownian motion of molecules belonging to the amorphous region, and referring to the molecular theory on the viscoelasticity of the completely amorphous polymer presented by Rouse and Bueche31, some motional modes associated with the cooperative motion of fairly large length of molecules, which will not be 


\section{MOTOWO TAKAYANAGI}

included within the lamellar phase, should be considered at the long time side or the high temperature side of the transition region of the crystalline polymer.

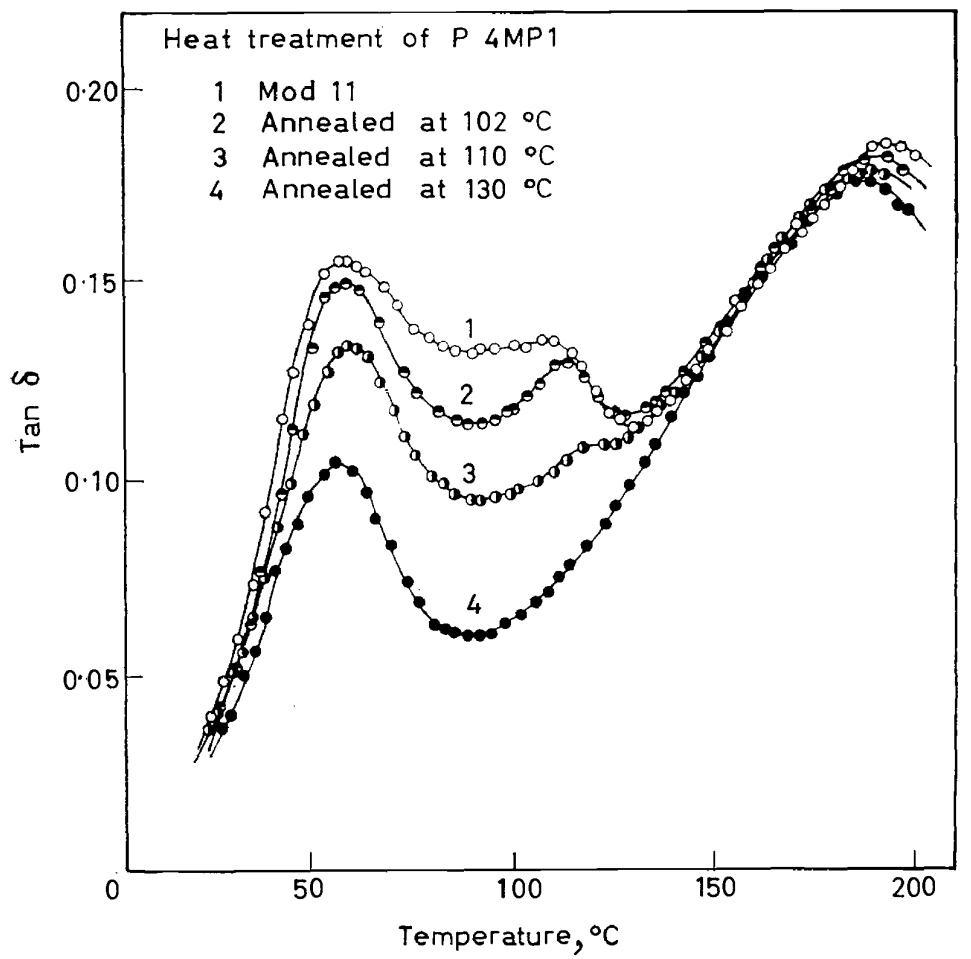

Figure 17. Tan $\delta$ vs. temperature at $110 \mathrm{c} / \mathrm{s}$ for the solution grown crystal mat of poly-4-methylpentene-1 and the samples annealed of it. The primary absorption $\left(\alpha_{\mathrm{a}}\right)$ is found at about $50^{\circ} \mathrm{C}$. The $120^{\circ} \mathrm{C}$ peak is related to the unstable crystal form.

For the polymers being almost absent from the primary absorption such as linear polyethylene or polyoxymethylene, the prediction that the amorphous region accords with the defect region within the lamellar phase may be allowed. However, for the polymers displaying the definite primary absorptions such as polyamide, polyester and poly- $\alpha$-olefins, it should be considered that the amorphous region associated with the primary absorption is segregated from the lamellar phase. In this view the single crystal of linear polyethylene prepared from xylene solution, in which the primary absorption is absent ${ }^{2}, 3$, will be composed of the molecules, being sharply folded at the crystal surface to form tight loops, which are incapable of micro-Brownian motion.

At this stage it will be necessary to present an example of single crystal displaying the primary absorption, which suggests the possibility of formation of loose loops attached to the crystal surface. Single crystal of trans-1,4polybutadiene (PBD) can be prepared from various solutions ${ }^{32}$. Figure 18 (given in the section printed on art paper) shows the electron photomicro- 
graph of single crystal of trans-1,4-polybutadiene prepared from benzene solution. Figure 19 shows that the dispersion curve of the mat of PBD single crystals (curve 2 ) has a remarkable primary absorption at about $-20^{\circ} \mathrm{C}$ at $110 \mathrm{c} / \mathrm{s}$, being largely different from the single crystal mats of polyethylene and polyoxymethylene. By annealing the mat of $\mathrm{PBD}$ crystals at $100^{\circ} \mathrm{C}$, the

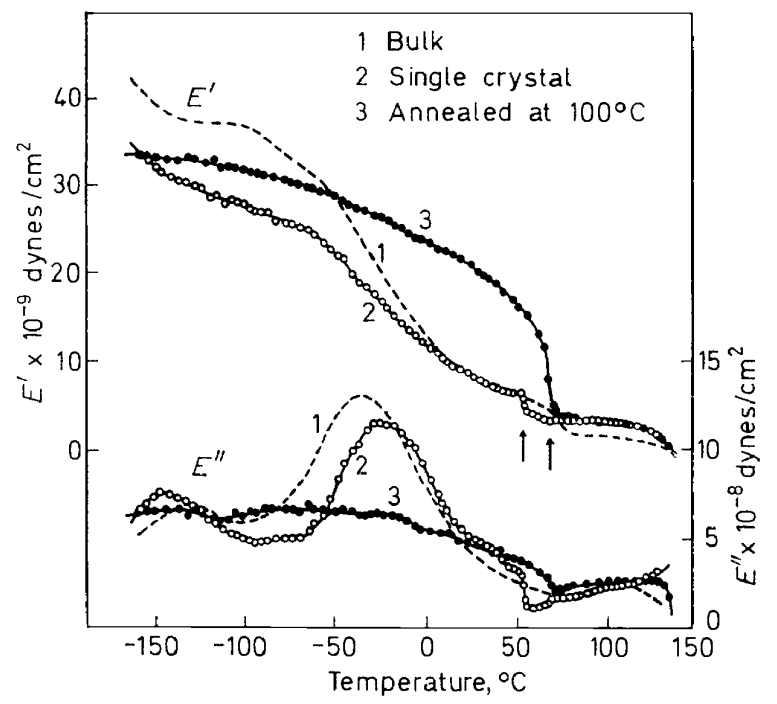

Figure 19. $E^{\prime}$ and $E^{\prime \prime}$ vs. temperature for the three kinds of samples of trans-1,4-polybutadiene at $110 \mathrm{c} / \mathrm{s}$ [(- - ) is for the bulk crystallized sample, $(O)$ is for the mat of single crystal, and (O) is for the single crystal mat annealed at $\left.100^{\circ} \mathrm{C}\right]$

$-20^{\circ} \mathrm{C}$ primary absorption has disappeared completely and the tensile storage modulus has been increased (curve 3 ). Dispersion curves expressed by the broken lines of Figure 19 are for the bulk crystallized samples (curve 1). Figure 20 shows the results of detailed inspection of the effect of annealing temperature on the primary absorption of PBD single crystal. In the results of Figure 20 the loose loops associated with the primary absorption disappear suddenly by annealing at $57^{\circ} \mathrm{C}$ just above the crystal transformation temperature $55^{\circ} \mathrm{C}^{33}$. The transformation temperature of the bulk crystallized $\mathrm{PBD}$ is known as $76^{\circ} \mathrm{C}$. The decrease of the crystal transformation temperature from $70^{\circ} \mathrm{C}$ to $55^{\circ} \mathrm{C}$ observed here is entirely caused by the morphological difference of lamella between the single crystal and the bulk crystallized sample. X-ray analysis proves that the crystal form of the single crystal sample changes from Form 1 to Form 2 at $55^{\circ} \mathrm{C}$ in the same way as the bulk crystallized one changes at $75^{\circ} \mathrm{C}$. At the crystal transformation temperature $55^{\circ} \mathrm{G}$ the lamellar thickness determined by the area method jumps from $100 \AA$ to $160 \AA$. Figure 21 shows the change of the crystal thickness evaluated by the line profile method applied to the meridional x-ray diffraction spot. The degree of crystallinity evaluated by the n.m.r. absorption method changes from 76 per cent of the single crystal mat to 91 per cent of the same sample annealed at $100^{\circ} \mathrm{C}$. The narrow component of n.m.r. differential curve is largely decreased by heat treatment. All these facts 


\section{MOTOWO TAKAYANAGI}

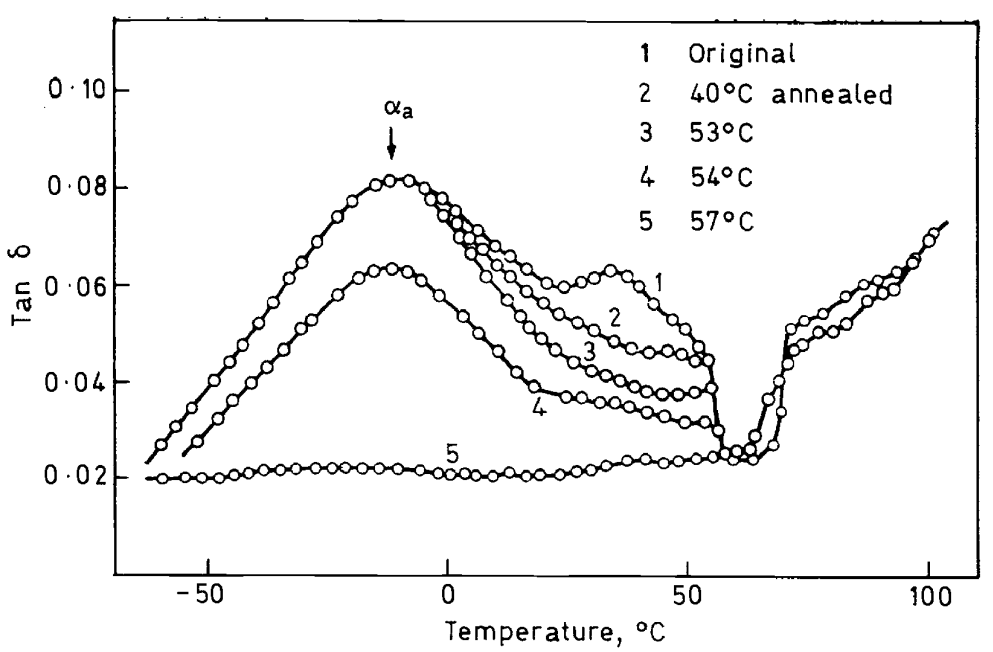

Figure 20. Effect of annealing temperature on the $\tan \delta \mathrm{vs}$. temperature curve at $110 \mathrm{c} / \mathrm{s}$ [Gurve 1 is for the original single crystal mat of trans-1,4-polybutadiene, curves $2,3,4$ and 5 are those for the single crystal mats annealed at $40^{\circ}, 53^{\circ}, 54^{\circ}$, and $57^{\circ} \mathrm{C}$ respectively. The crystal transformation temperature is $55^{\circ} \mathrm{C}$ ]

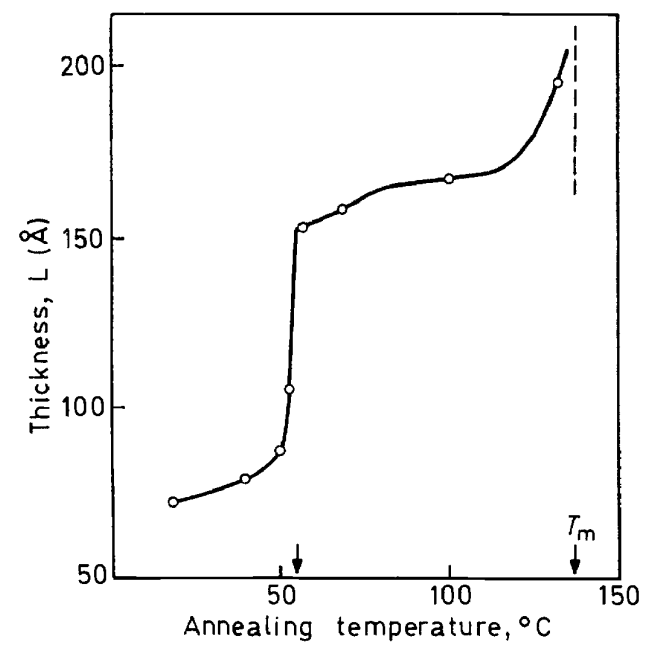

Figure 21. Thickness of crystal phase of single crystal of trans-1,4-polybutadiene vs. annealing temperature. Thickness is determined by the line profile method applied to the meridional $\mathrm{x}$-ray diffraction spot.

prove that the loose loops causing the primary absorption are attached directly to the single crystal surface and by heat treatment these loose loops are tightened, being accompanied at the same time by the thickening of the single crystal. Figure 22 schematically represents the models interpreting the phenomena of disappearance of the primary absorption and of thickening of lamellae. In Figure 22 the lamellar thickness was evaluated by comparison of the area of the single crystal domain before and after heat treat- 


\section{GRYSTALLIZED STATE OF POLYMER IN ITS DISPERSION BEHAVIOUR}

ment in the electron photomicrographs. The thickness of crystalline region of the original single crystal $72 \AA$ was evaluated by the line profile of the $x$-ray diffraction spot near the meridian corrected for the instrumental broadening. The crystallinity of the original sample evaluated by the values of the total and the crystal portion thickness is 74 per cent after correction
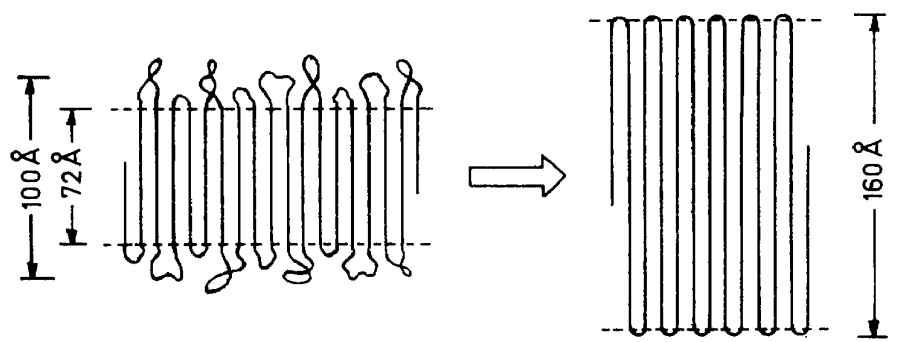

Figure 22. Schematic representation of thickening process of single crystal of trans-1,4-polybutadiene with loose loops at the crystal transformation temperature. Lamellar thickness is determined by the area method and crystal thickness is determined by the line profile method.

of density and accords well with the value of 76 per cent evaluated by n.m.r. absorption.

It is interesting that the average length of loose loops in the original crystal is evaluated from the above data as about $28 \AA$ or 22 carbon atoms and this length is considered to be enough to initiate the micro-Brownian motion.

In order to survey the effect of the solvent power on the primary absorption of PBD single crystal it will be meaningful to discuss the mechanism of loose loop formation. Figure 23 shows the tan $\delta$ curves of single crystal mats prepared from benzene, cyclohexane, n-heptane and methyl isobutyl ketone (MIBK) at the crystallization temperatures of $18^{\circ}, 24^{\circ}, 62^{\circ}$ and $75.5^{\circ} \mathrm{C}$ respectively ${ }^{32}$. The intensity of the primary absorption in Figure 23 is increased in the order of MIBK, n-heptane, cyclohexane and benzene, i.e. with increasing solvent power. The solvent power was judged by the magnitude of the melting point depression according to Flory's equation ${ }^{34}$. It predicts that the melting point depression by the solvent is determined by the solvent-polymer interaction parameter if the molar volume of the solvent is not so much different among the solvents used. A good solvent largely depresses the melting temperature as compared with a poor solvent.

A possible interpretation of the above results is that the free energy of formation of the critical nucleus for growth will be decreased, if the loops interact more with good solvents than with poor solvents to make loops loosen. Poor solvent has, on the other hand, a tendency of reducing the contact with the polymer segments to form rather tightened loops. Figure 24 shows a schematic representation of hypothetical growth mechanism with loose and tight loops in good and poor solvents respectively.

It is known that the primary absorption of bulk crystallized polymers does not disappear by heat-treatment. The primary absorption of bulk crystallized trans-1,4-polybutadiene represented by the broken lines in Figure 19 did not 


\section{MOTOWO TAKAYANAGI}

disappear by heat-treatment at $100^{\circ} \mathrm{C}$, while the primary absorption of the single crystal mat of the same polymer easily disappeared by the same treatment. This observation suggests that the existence of tie molecules between neighbouring lamellae largely resists the change of the state of the amorphous region of the bulk crystallized samples.

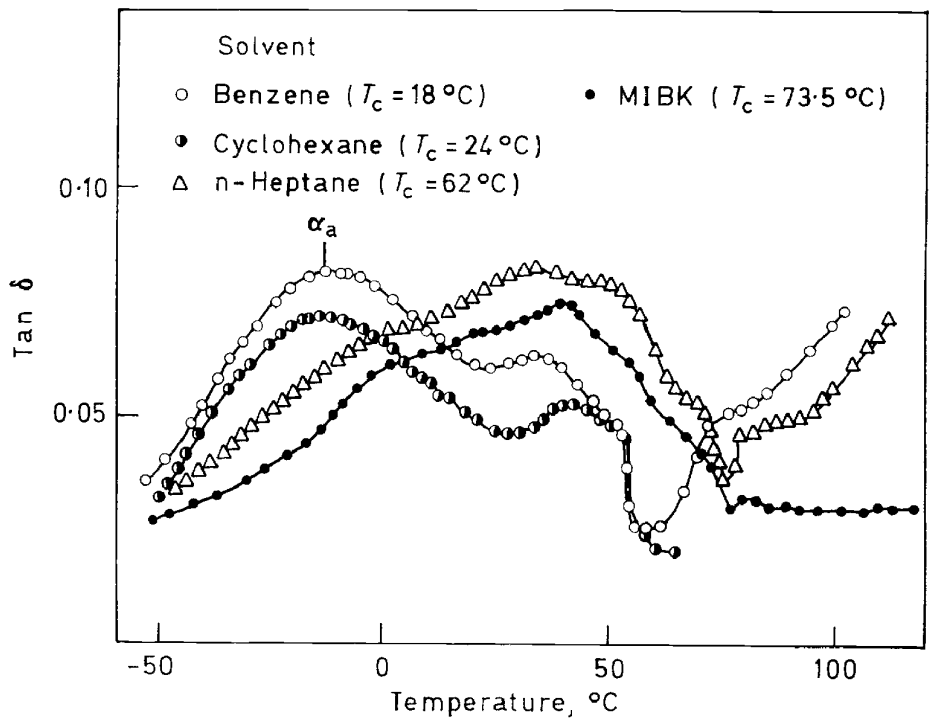

Figure 23. Effect of the solvents used to prepare the trans-1,4-polybutadiene single crystal upon the $\tan \delta$ vs. temperature curve at $110 \mathrm{c} / \mathrm{s}$. Benzene (O), cyclohexane (O), n-heptane $(\triangle)$, and methyl isobutylketone $(\bullet)$ are used at the crystallization temperatures, $18^{\circ}, 24^{\circ}$, $62^{\circ}$, and $73 \cdot 5^{\circ} \mathrm{C}$ respectively. Notice the $\tan \delta$ peak at about $-20^{\circ} \mathrm{C}$ corresponding to the primary absorption

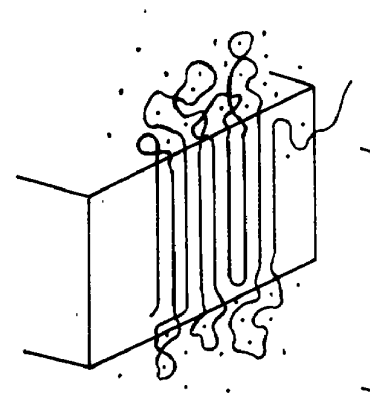

Good solvent

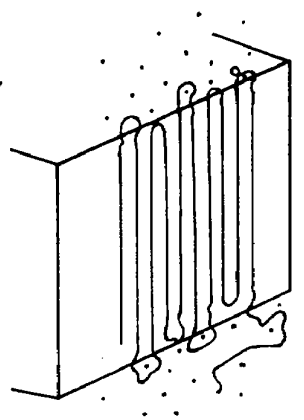

Poor solvent

Figure 24. Schematic representation of hypothetical growth mechanisms with loose and tight loops in good and poor solvents

The model of spherulite in its detailed structure presented by the authors 18 has already been shown in Figure 9. Palmer and coworkers ${ }^{35}$ have first shown that the polyethylene spherulite gives well-defined crystal plates like single crystal after etching with fuming nitric acid. A similar experiment 
has recently been tried on the bulk crystallized sample of isotactic polypropylene by Minami ${ }^{36}$, who treated this sample with fuming nitric acid and measured the viscoelastic properties of the etched residues embedded in the cellulose acetate. Figure 25 shows that the remarkable primary absorption

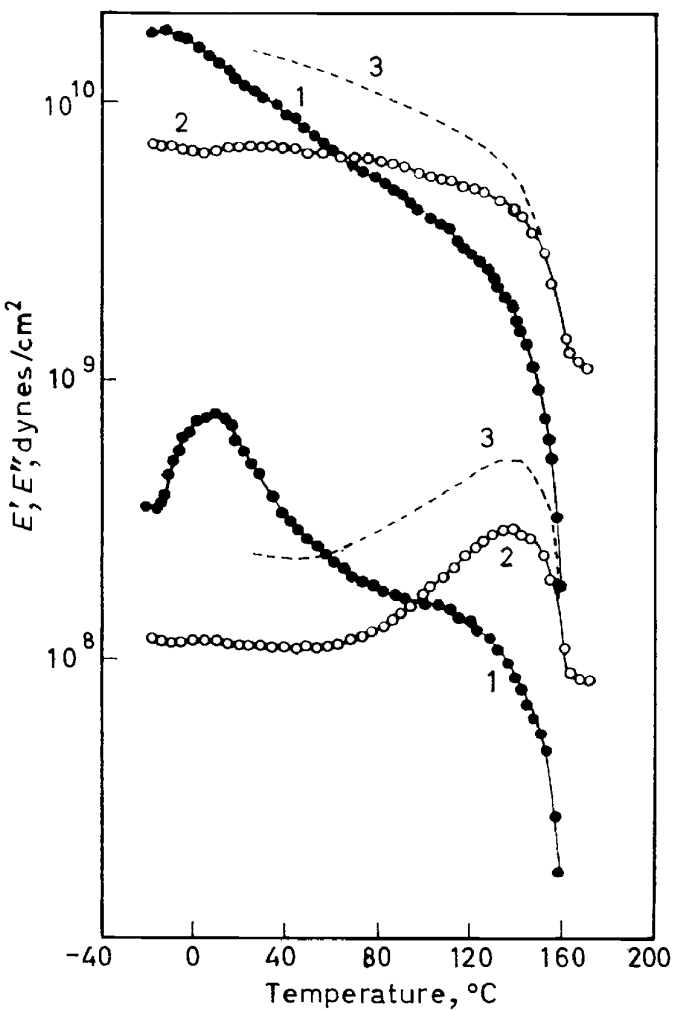

Figure 25. Effect of etching with fuming nitric acid on bulk crystallized isotactic polypropylene (Minami $\left.{ }^{36}\right)[(\mathbf{O})$ is for the sample untreated, $(O)$ is for the sample etched by nitric acid, and $(---)$ is for the mat of solution grown crystals prepared from xylene]

of the original sample almost disappeared and the small $120^{\circ} \mathrm{C}$ crystalline peak of the original sample was overwhelmingly increased after nitric acid treatment. It is interesting that the absorption curve of the etched sample shows almost the same shape as that of the solution-grown crystal of polypropylene.

The crystallized state of polymer will be clarified in more detail if a knowledge of molecular motion from dispersion behaviour is added to the information from the structural and morphological studies.

\section{DISPERSION GURVES AND HIGHER ORDER STRUCTURE}

Higher order structure of the bulk crystallized polymer is considered to be composed of the spherulitic lamellae and the amorphous region filling interlamellar space. Above the glass transition temperature the bulk crystallized sample resembles in some point high impact plastics, which are 


\section{MOTOWO TAKAYANAGI}

composed of rubber particles dispersed in the matrix of hard plastics. The high impact interpolymer resin shows the rubber absorption at the low temperature and the plastics absorption at the high temperature of its dispersion curve. In the bulk crystallized polymer the primary absorption and the crystalline absorption can be observed in its dispersion curve, as if the amorphous region and the crystalline region correspond to the rubber and to the plastics phase of the interpolymer respectively. The way of mechanical connection of the amorphous and the crystalline region can be revealed by a detailed inspection of the dispersion curve. Such a model for the bulk crystallized polymer was discussed in the previous papers ${ }^{2,37}$, where the dispersion behaviour of the specifically constructed model fairly well reproduced that of the crystallized polymer with various degrees of crystallinity. In this paper we will apply similar considerations to the oriented polymers ${ }^{38}$.

Crystallized samples of polyethylene (PE), isotactic polypropylene $(\mathbf{P P})$, polytetrahydrofuran (PTHF), polyethylene oxide (PEO) and polyoxymethylene (POM) were well annealed after an orientation was given by drawing or rolling. The higher order structure of these samples were surveyed by using $x$-ray small angle scattering. From the intensity distribution along equatorial, layer line or meridian, it was concluded that the microfibrils of these samples are united by heat-treatment to form huge fibrils with large diameter, and that the arrangement of the crystalline region $(C)$ and the amorphous region $(A)$ becomes regular in the way that $A$ and $C$ align in series along the fibre axis and in parallel along the direction perpendicular to the fibre axis. Figure $26(a)$ shows the Hosemann's model ${ }^{39}$ for the hot-drawn polyethylene. This model can be reasonably applied to the

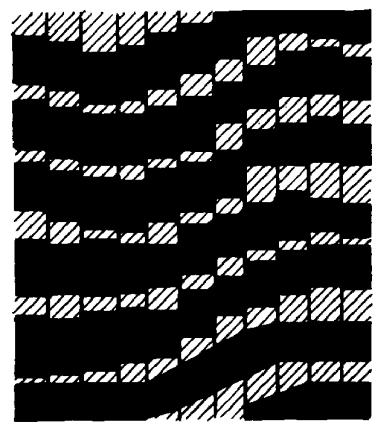

(a)

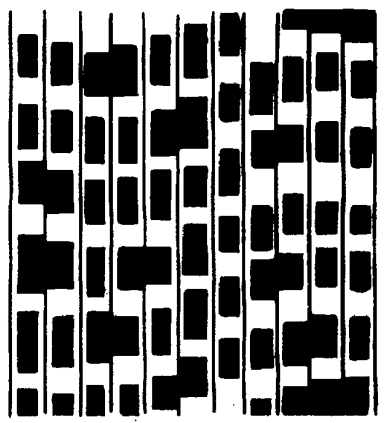

(b)

Figure 26. (a) Structural model for hot-stretched linear polyethylene presented by Hosemann $^{39}$. (b) Slightly modified Hosemann's model, which interprets the actual dispersion behaviours of the drawn and annealed polymers [Amorphous regions are expressed by black areas]

higher order structure of the other samples used here in view of the results of small angle x-ray scattering.

There is a general relationship common to all dispersion curves inspected here, i.e. those of the annealed oriented PE, PP, POM, PEO and PTHF, 


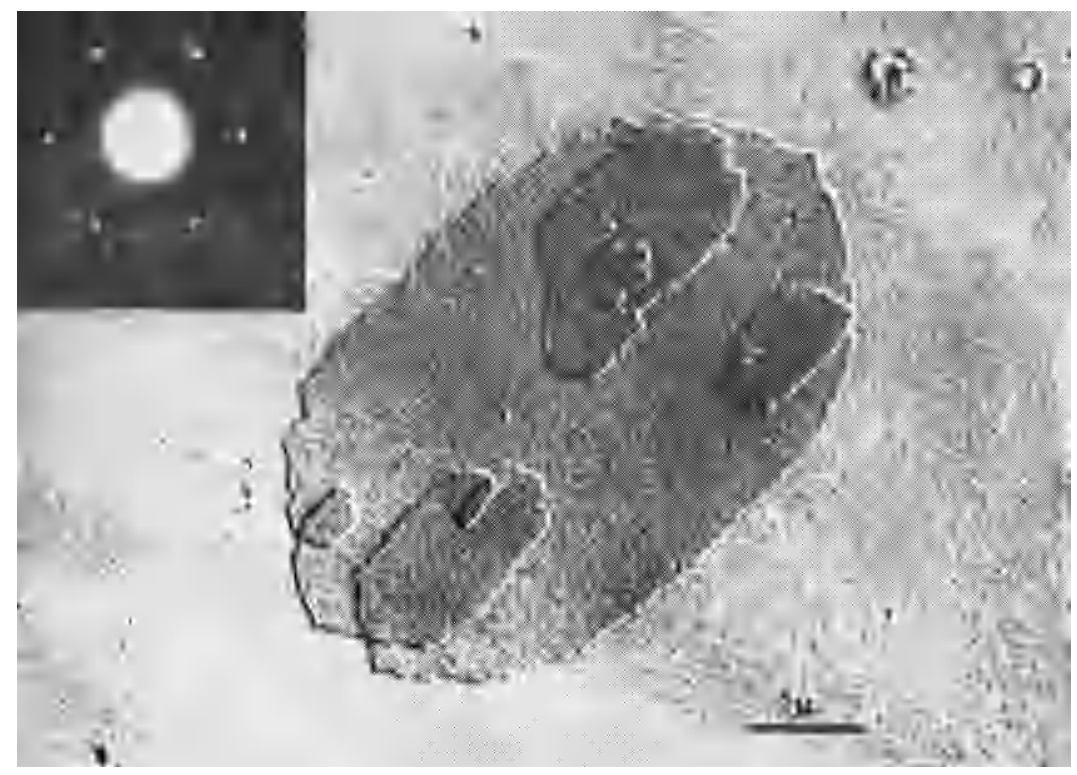

Figure 18. Electron photomicrograph of single crystal of trans-1,4-polybutadiene prepared from benzene solution at $18^{\circ} \mathrm{C}$ and its electron diffraction pattern 


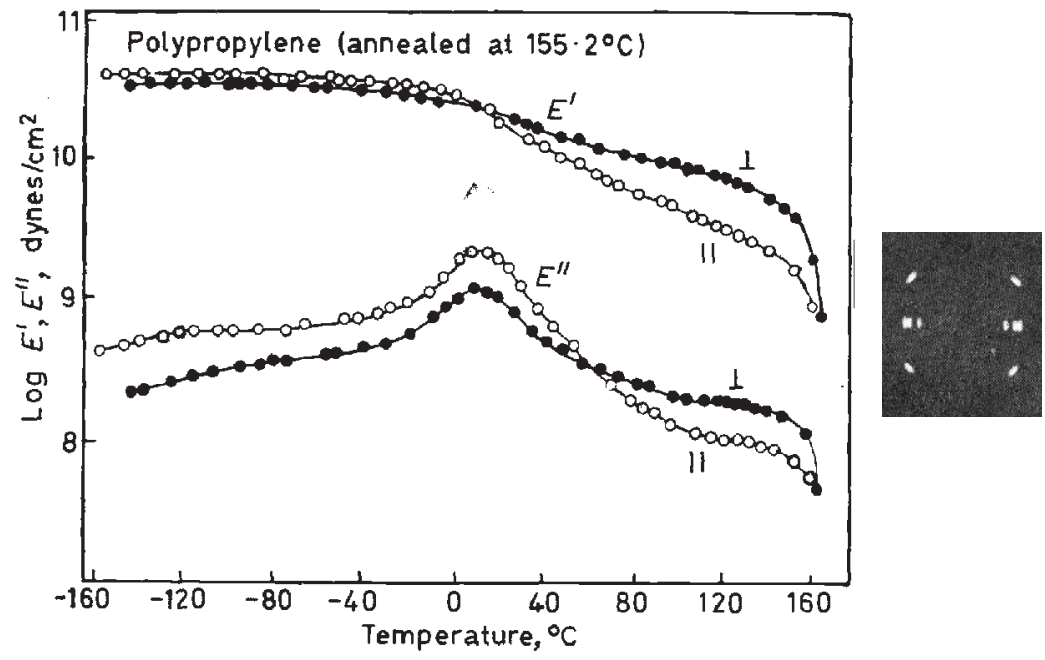

Figure 27. Temperature dependence of $E^{\prime}$ and $E^{\prime \prime}$ along and perpendicular to the draw direction for the annealed samples of drawn polypropylene at $110 \mathrm{c} / \mathrm{s}$. X-ray fibre diagram is for the same sample.

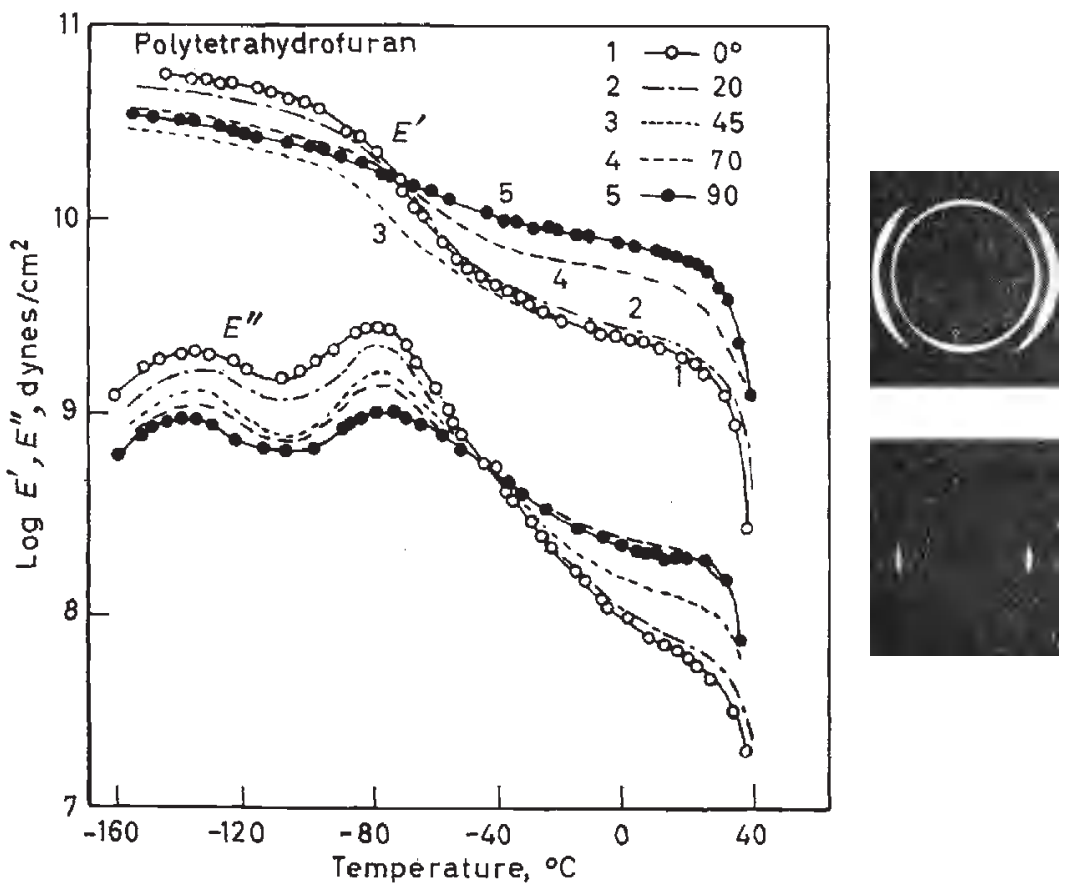

Figure 28. Temperature dependence of $E^{\prime}$ and $E^{\prime \prime}$ in directions of $0,20,45,70$ and $90^{\circ}$ to the draw direction for the annealed samples of drawn polytetrahydrofuran [The upper x-ray diffraction diagram is obtained with $\mathrm{x}$-ray beam parallel to the drawing direction and the lower diagram is obtained with $\mathrm{x}$-ray beam perpendicular to the draw direction (fibre diagram)] 


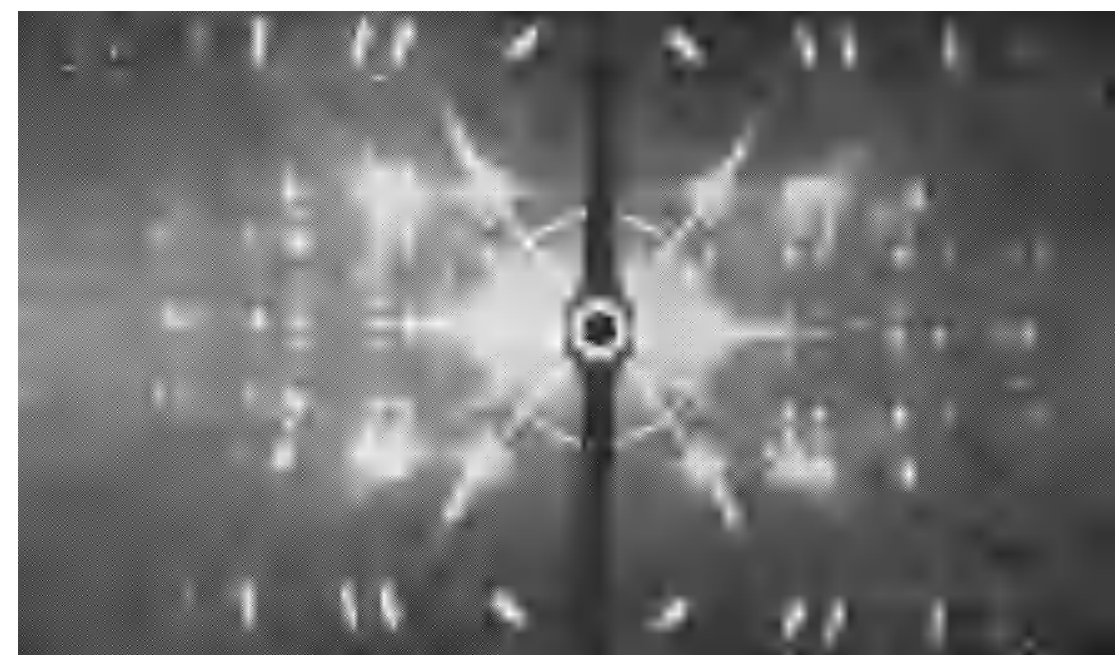

Figure 33. Rotational $x$-ray diffraction diagram of polytetraoxane crystal polymerized in a solid state by gamma-ray irradiation used for the viscoelastic measurements 


\section{GRYSTALLIZED STATE OF POLYMER IN ITS DISPERSION BEHAVIOUR}

that the modulus (tensile storage modulus at $110 \mathrm{c} / \mathrm{s}$ ) along the fibre axis is higher than that of the perpendicular direction below the primary absorption temperature, they intersect at this temperature and above that temperature the modulus along the fibre axis becomes lower than the perpendicular one. Figures 27 and 28 (see art section) show the dispersion curves along and perpendicular to the draw direction for drawn annealed $\mathrm{PP}$ and PTHF respectively. X-ray diffraction patterns of the same samples are cited together. These are two examples of the general phenomena of modulus intersection. The primary absorptions are not distinct of linear polyethylene and polyoxymethylene and their intersecting temperatures are not clear, but they are considered to be located at around the temperatures of the latent primary absorptions 16,40 .

The general relationship observed here will be qualitatively understood by adopting the Hosemann's model Figure 26 (a) for the well annealed oriented polyethylene and by taking into account both the anisotropy of the polymer crystal and the approximately isotropic amorphous region. Figure 29 shows the schematic representation of the mechanical model corresponding

(a)
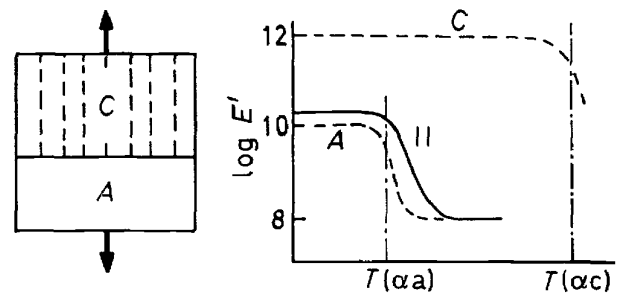

(b)

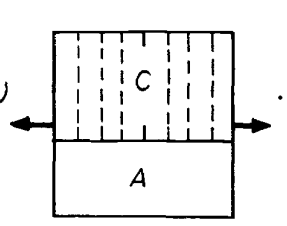

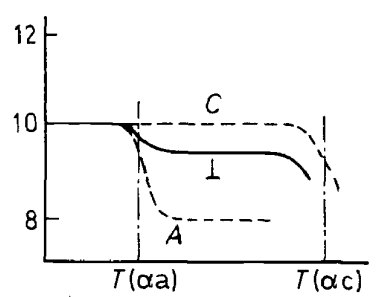

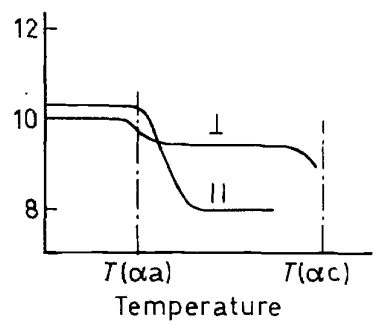

Figure 29. Schematic representations of dispersion curves of $E^{\prime}$ (solid lines) in the parallel direction $(a)$ and the perpendicular direction $(b)$ to the draw direction. Calculations are made by use of the $E^{\prime}$ values of the amorphous $(A)$ and the crystalline region $(C)$ as expressed by the broken lines. Part $(c)$ represents the difference between the dispersion curves of the parallel direction and the perpendicular direction. Directions of applied force are indicated by the arrows in the model, which is a simplified one of Figure $26(a)$. 


\section{MOTOWO TAKAYANAGI}

with the Hosemann's model of Figure $26(a)$. The modulus of the crystalline region was assumed to be $10^{12} \mathrm{dyne} / \mathrm{cm}^{2}$ for the parallel direction and $10^{10}$ dyne $/ \mathrm{cm}^{2}$ for the perpendicular direction, and the modulus of the amorphous region was assumed to be $10^{10}$ dyne $/ \mathrm{cm}^{2}$ below the glass transition temperature and $10^{8}$ dyne $/ \mathrm{cm}^{2}$ above the glass transition temperature. Figure 29 also shows the dispersion curves calculated for the cases when the force is applied in parallel and perpendicular to the draw direction. The model presented here is successful in qualitative explanation of the intersection of modulus, but as compared with the experimental results it is more reasonable to modify the $A C$ series model like the one shown in Figure $26(b)$. The modified model of Figure $26(b)$ is improved in interpreting that the crystalline absorption can be clearly found along the draw direction and the modulus along the draw direction above $T_{\mathrm{g}}$ does not decrease as much as expected from the simple $A C$ series model. The modified model is composed of the laterally developed and oriented lamellar crystals, thin oriented crystals connecting these lamellae along the draw direction, and the inter-lamellar amorphous regions.

X-ray small angle scattering of the cold-drawn samples reveals the broad distribution of the microfibril diameter and the spatially irregular arrangement of the amorphous and the crystalline region. The modulus perpendicular to the fibre axis is expected to be largely decreased by the presence of microvoids between the microfibrils. The left-hand side of Figure 3

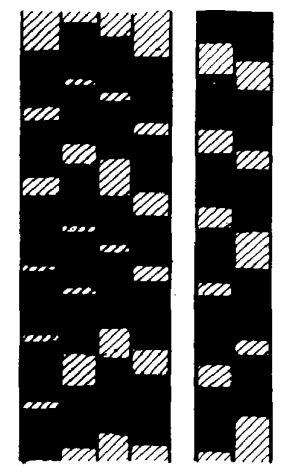

(a)

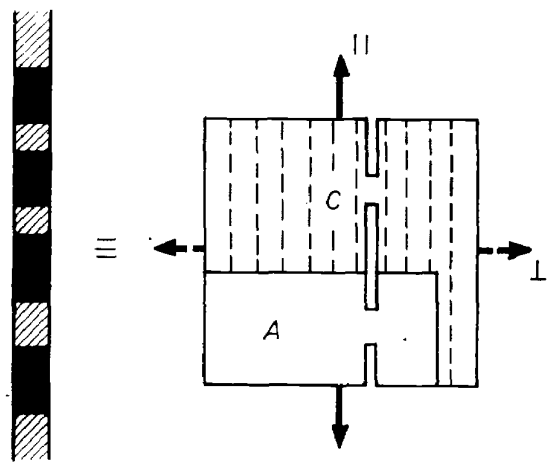

(b)

Figure 30. (a) Structural model of cold-drawn linear polyethylene presented by Hosemann ${ }^{39}$, which is composed of splitted microfibrils with different diameters. $(b)$ Mechanical model corresponding with the structural model in $(a)$, expressing the various ways of response depending on the direction of applied force.

the model reproduced from Hosemann's paper ${ }^{39}$, which is given for the cold-drawn polyethylene. The right-hand side of Figure 30 is the corresponding mechanical model. Actually in the dispersion curves of colddrawn linear polyethylene and polypropylene there could not be found any intersection of the moduli along the draw direction with that along the perpendicular direction as shown in Figures 31 and 32. Detailed inspection of the value of modulus leads us necessarily to adopt the anisotropy of the 


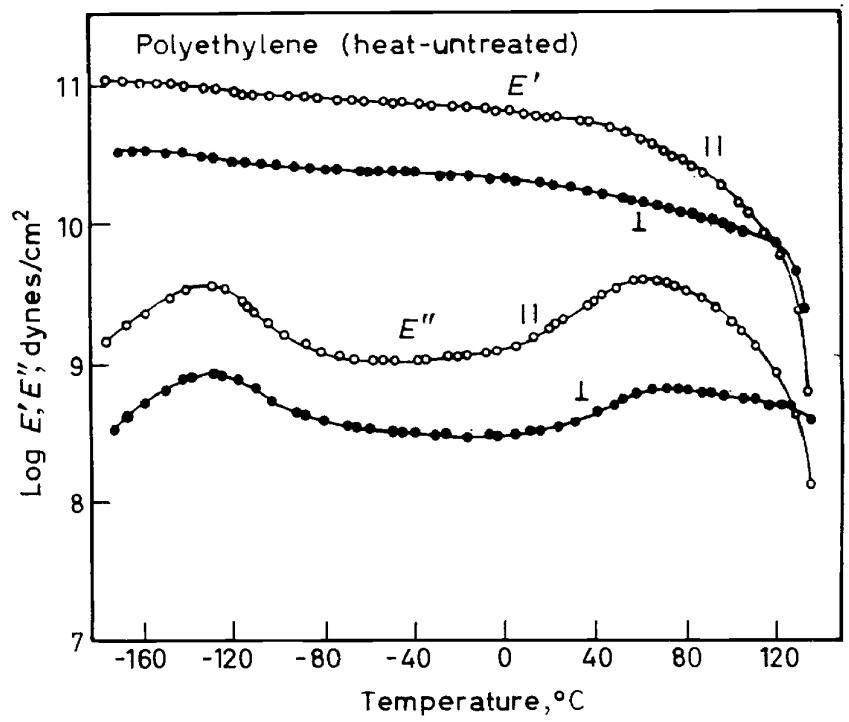

Figure 31. Temperature dependence of $E^{\prime}$ and $E^{\prime \prime}$ along and perpendicular to the drawing direction for the cold-drawn film of linear polyethylene at $110 \mathrm{c} / \mathrm{s}$

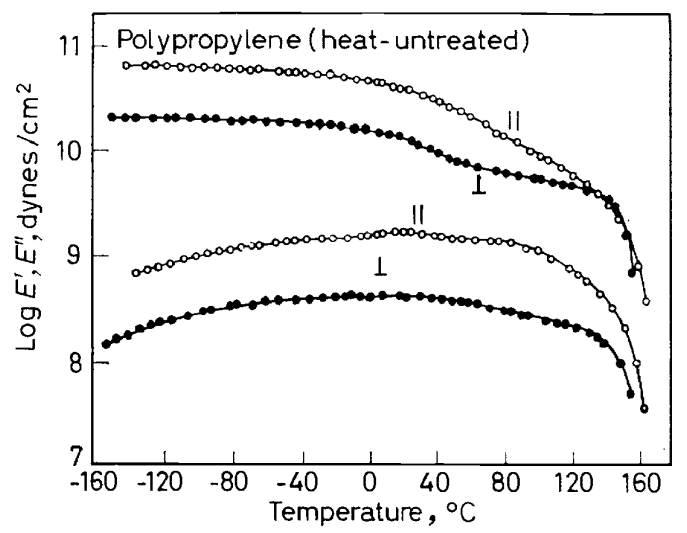

Figure 32. Temperature dependence of $E^{\prime}$ and $E^{\prime \prime}$ along and perpendicular to the drawing direction for the cold-drawn film of isotactic polypropylene at $110 \mathrm{c} / \mathrm{s}$

amorphous region, which is caused by the insufficient relaxation of the oriented amorphous region due to low drawing temperature.

In order to understand the higher order structure of the industrial fibres or oriented films it would be very instructive to know the dispersion behaviour of the ideally oriented polymer crystal with the highest perfection. Single crystal of tetraoxane can be polymerized in a solid state by gammaray irradiation to form the polymer crystal composed of extended chains of polyoxymethylene ${ }^{41}$. Figure 33 shows the rotational x-ray diffraction pattern of polytetraoxane crystal, which proves that molecular chains of polyoxymethylene are ideally oriented along the long axis of the crystal. 


\section{MOTOWO TAKAYANAGI}

After extraction of residual monomer the tensile viscoelastic measurements were made along the long axis of the crystal. Figure 34 shows the $\tan \delta$ ss. temperature curve for polytetraoxane (curve 1), polyoxymethylene fibre prepared by drawing the crystallized polymer (curve 2) and single crystal mats of polyoxymethylene (curve 3 ). Tan $\delta$ peak at about $-70^{\circ} \mathrm{C}$ corresponds to the secondary absorption and its mechanism of relaxation is

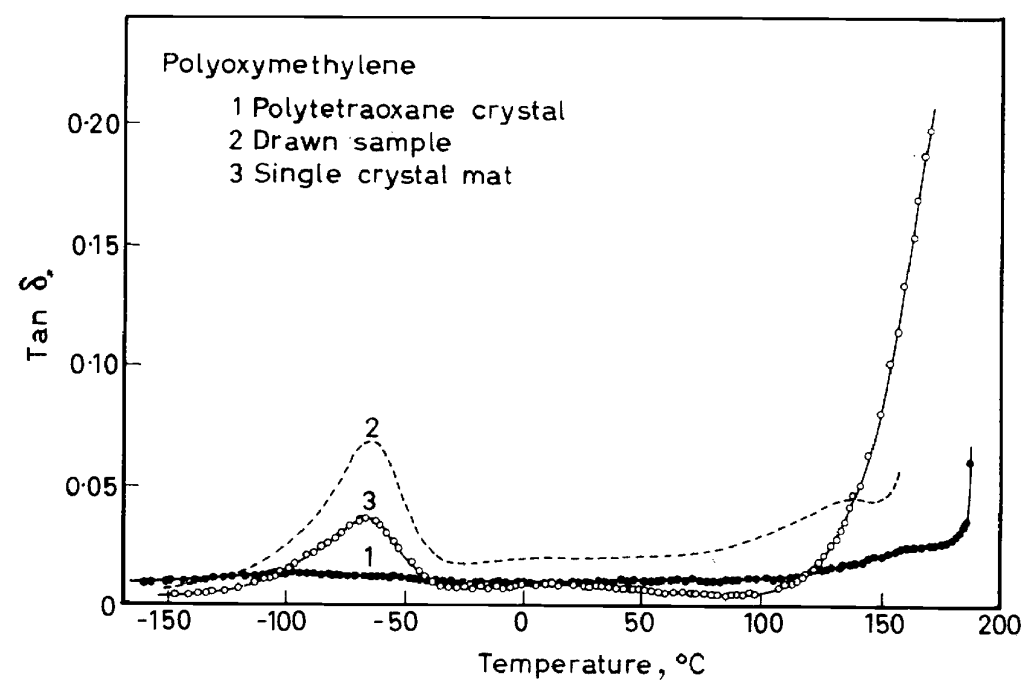

Figure 34. Tan $\delta \mathrm{vs}$. temperature curves for three kinds of samples of polyoxymethylene [(O) is for the solid-state polymerized polytetraoxane crystal along its molecular chain direction, (- - ) is for the fibre sample prepared by drawing the crystallized polymer along the draw direction, and $(O)$ is for the single crystal mat of polyoxymethylene along the mat surface]

ascribed to the local twisting motion of main chains in the defect region of crystal and in the frozen amorphous region 16,18 . The fact that there is no secondary absorption in polytetraoxane crystal as shown in Figure 34 predicts that the polytetraoxane crystal is almost perfect, being absent from defect region. The single crystal sample (curve 3 ) in Figure 34 includes some such defect regions and the so-called fibre prepared by drawing (curve 2) has an unexpectedly large number of defects in its structure. Figure 35 shows the tensile storage modulus and the tensile loss modulus of the three samples as a function of temperature. The storage modulus of polytetraoxane displays an outstanding constancy of the modulus value very close to the melting temperature $190^{\circ} \mathrm{C}$, while the modulus value of drawn polyoxymethylene (broken lines) decreases gradually from about the secondary absorption temperature, suggesting many kinds of relaxation mechansisms associated with the various types of disorder. Referring to the dispersion curves of the three samples, the drawn fibre rather resembles in some point the single crystal sample. This suggests that the lamellar structure composed of folded molecules has a possibility of being included in the oriented fibre. To prove it, however, it is necessary to have more information on fibre structure. 


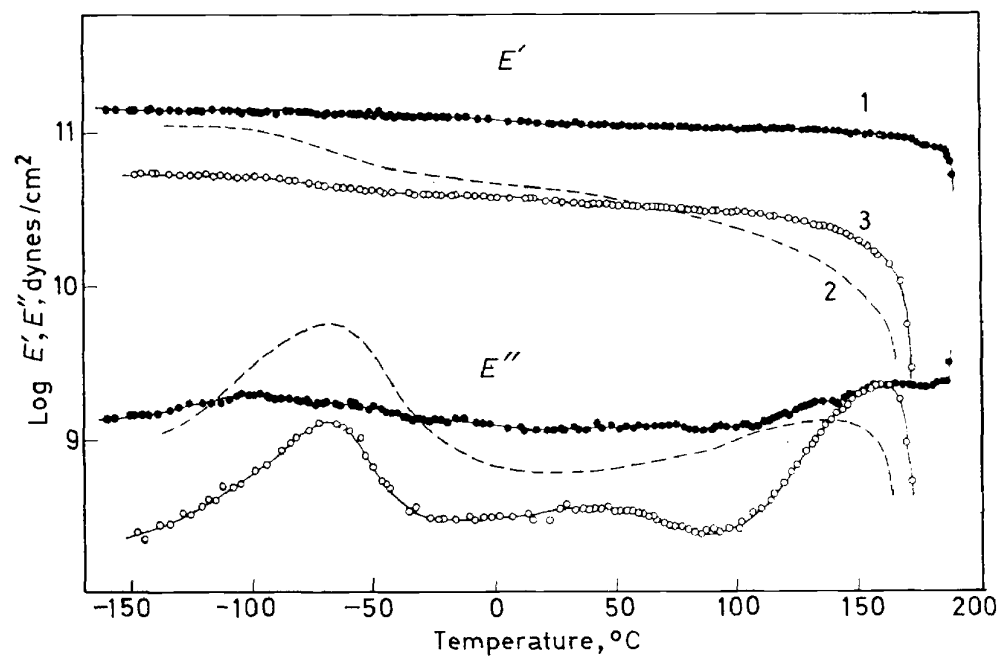

Figure 35. $E^{\prime}$ and $E^{\prime \prime}$ vs. temperature for three kinds of samples of polyoxymethylene $[(\boldsymbol{O})$ is for the polytetraoxane crystal, $(---)$ is for the drawn sample, and $(O)$ is for the single crystal mat. These samples are the same as in Figure 34]

Measurement of viscoelastic absorption is one of the methods of obtaining the information on molecular motions. The dielectric absorption and the broad line n.m.r. absorption are also available. The purpose of this article is to draw attention to the fact that the information from dispersion behaviour is useful to approach to the true features of the crystallized polymer by combined use of the morphological and of the structural methods.

\section{References}

1 A. Keller. Phil. Mag. 2, 1171 (1957).

2 M. Takayanagi. Proceedings of the Fourth International Congress on Rheology, Part 1, pp. 167-187, Interscience Publishers, New York, 1965.

3 M. Takayanagi. Kobunshi, 14, 314 (1965); J. Soc. Material Science, Japan, 14, 343 (1965).

4 M. Yoshino and M. Takayanagi. J. Japan Soc. Test. Mat. 6, 330 (1959).

5 M. Takayanagi, K. Imada, A. Nagai, T. Tatsumi and T. Matsuo, papers presented at the International Symposium on Macromolecular Chemistry, Aug. 1965, Prague; J. Polymer Sci. C. (16), 867 (1967).

6 K. Okano. Rikenhokoku 5, 273, 295 (1964).

7 J. I. Lauritzen, Jr. and J. D. Hoffman. J. Res. Nat. Bur. Standards, in the press.

8 M. G. Broadhurst. J. Chem. Phys. 36, 2578 (1962).

9 V. F. Holland. J. Appl. Phys. 35, 3234 (1964).

10 R. W. Kedzie, cited in Polymer Single Crystals (by P. H. Geil), Interscience Publishers, New York, 1963, p. 337.

11 W. P. Slichter. J. Appl. Phys. 31, 1865 (1961).

12 S. Iwayanagi and I. Miura. Japanese J. Appl. Phys. 4, 94 (1965).

13 K. M. Sinnott. J. Appl. Phys. 37, 3385 (1966).

14 J. D. Ferry. Viscoelastic Properties of Polymers, John Wiley \& Sons, Inc., New York, 1960, Chapter 3.

15 M. Takayanagi and T. Matsuo, paper presented at the International Symposium on Macromolecular Chemistry, Tokyo-Kyoto, 1966; J. Macromol. Sci. (Phys.) B1(3), 407 (1967).

16 M. Takayanagi. Mem. Fac. Engineering, Kyushu Univ., 23, 1 (1963).

17 M. Takayanagi and N. Kawasaki. J. Macromol, Sci. (Phys.) B1(4), 741 (1967).

18 M. Takayanagi, M. Yoshino and S. Minami. J. Polymer Sci. 61, S7 (1962).

10 K. Yamafuji. J. Phys. Soc. Japan 15, 2295 (1960).

20 K. H. Illers and H. Breuer. J. Colloid Sci. 18, 1 (1963).

21 Y. Ishida, K. Yamafuji, H. Ito and M. Takayanagi. Kolloid-Z. u. Z. Polymer 184, 97 (1962). 


\section{MOTOWO TAKAYANAGI}

22 Y. Ishida, H. Ito and M. Takayanagi. Polymer Letters 3, 87 (1965).

${ }^{23}$ H. Yasuda, Y. Tanda and M. Takayanagi. J. Chem. Soc. Japan, Ind. Chem. Sect. 69, 304 (1966).

24 A. Nagai and M. Takayanagi. J. Chem. Soc. Japan., Ind. Chem. Sect. 68, 836 (1965).

$25 \mathrm{~K}$. Neki and M. Takayanagi. Reports on Progress in Polymer Physics in Japan 8, 281 (1965).

${ }^{26}$ G. Natta. Makromol. Chem. 35, 93 (1960).

27 S. Minami, Y. Tanoue and M. Takayanagi. J. Chem. Soc. Japan, Ind. Chem. Sect. 68, 830 (1965).

28 Y. Tanda, K. Imada, and M. Takayanagi. J. Chem. Soc. Japan, Ind. Chem. Sect. 69, 1971 (1966).

$29 \mathrm{H}$. Nakamura, S. Manabe and M. Takayanagi, unpublished work.

${ }^{30} \mathrm{~A}$. Nagai and T. Ishibashi and M. Takayanagi, unpublished work.

31 cf. J. D. Ferry, Viscoelastic Properties of Polymers, John Wiley \& Sons, New York, 1961, p. 151.

${ }^{32} \mathrm{~S}$. Takamuku, T. Tatsumi, K. Imada and M. Takayanagi, paper presented at the Annual Meeting of the Society of Polymer Science, Japan, May 22, 1966.

33 T. Tatsumi, T. Fukushima, K. Imada, and M. Takayanagi, paper presented at the International Symposium on Macromolecular Chemistry, 1966, at Tokyo and Kyoto; J. Macromol. Sci. (Phys.), B1(3), 459 (1967).

34 P. J. Flory, Principles of Polymer Chemistry, Cornell Univ. Press, New York, 1953, p. 568.

35 R. P. Palmer and A. J. Cobbold. Makromol. Chem. 74, 174 (1964).

$36 \mathrm{~S}$. Minami, private communication.

${ }^{37}$ M. Takayanagi, S. Uemura and S. Minami. J. Polymer Sci., Part C. 5, p. 113 (1963).

${ }^{38}$ M. Takayanagi, K. Imada and T. Kajiyama, paper presented at the Joint U.S.-Japan Polymer Physics Seminar at Kyoto, Oct. 20. 1965. J. Polymer Sci., C(15), 263 (1966).

39 R. Hosemann. J. Appl. Phys. 34, 25 (1963).

40 L. Bohn. Kolloid-Z. 201, 20 (1965).

${ }^{41}$ K. Hayashi, H. Ochi, M. Nishii, Y. Miyake and S. Okamura. Polymer Letters 1, 427 (1963). 